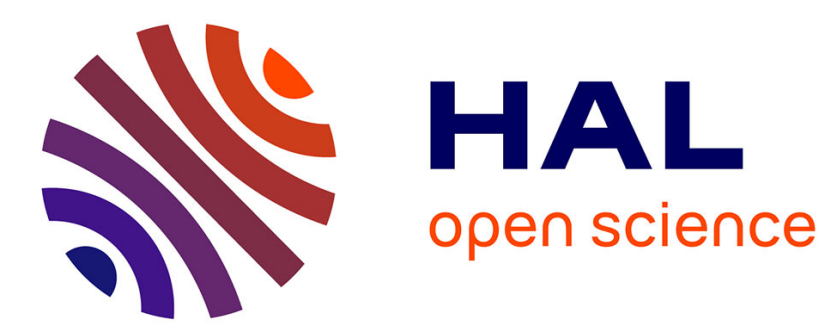

\title{
An injective version of the 1-2-3 Conjecture
}

Julien Bensmail, Bi Li, Binlong Li

\section{To cite this version:}

Julien Bensmail, Bi Li, Binlong Li. An injective version of the 1-2-3 Conjecture. Graphs and Combinatorics, 2021, 37, pp.281-311. 10.1007/s00373-020-02252-y . hal-02459377

\section{HAL Id: hal-02459377 https://hal.science/hal-02459377}

Submitted on 29 Jan 2020

HAL is a multi-disciplinary open access archive for the deposit and dissemination of scientific research documents, whether they are published or not. The documents may come from teaching and research institutions in France or abroad, or from public or private research centers.
L'archive ouverte pluridisciplinaire HAL, est destinée au dépôt et à la diffusion de documents scientifiques de niveau recherche, publiés ou non, émanant des établissements d'enseignement et de recherche français ou étrangers, des laboratoires publics ou privés. 


\title{
An injective version of the 1-2-3 Conjecture*
}

\author{
Julien Bensmail ${ }^{1}, \mathrm{Bi} \mathrm{Li}^{2}$, and Binlong $\mathrm{Li}^{3}$ \\ ${ }^{1}$ Université Côte d'Azur, CNRS, Inria, I3S, France \\ ${ }^{2}$ Xidian University, Xi'an, China \\ ${ }^{3}$ Northwestern Polytechnical University, Xi'an, China
}

January 27, 2020

\begin{abstract}
In this work, we introduce and study a new graph labelling problem standing as a combination of the 1-2-3 Conjecture and injective colouring of graphs, which also finds connections with the notion of graph irregularity. In this problem, the goal, given a graph $G$, is to label the edges of $G$ so that every two vertices having a common neighbour get incident to different sums of labels. We are interested in the minimum $k$ such that $G$ admits such a $k$-labelling.

We suspect that almost all graphs $G$ can be labelled this way using labels $1, \ldots, \Delta(G)$. Towards this speculation, we provide bounds on the maximum label value needed in general. In particular, we prove that using labels $1, \ldots, \Delta(G)$ is indeed sufficient when $G$ is a tree, a particular cactus, or when its injective chromatic number $\chi_{\mathrm{i}}(G)$ is equal to $\Delta(G)$.
\end{abstract}

\section{Introduction}

We deal with undirected graphs only. By a labelling $\ell$ of some graph $G$, we mean a mapping $\ell: E(G) \rightarrow L$ assigning labels to the edges of $G$ (from a set $L$ of labels). For every vertex $v$ of $G$, we can compute the sum of the labels on its incident edges, and assign this value as the colour $c_{\ell}(v)$ of $v$. Doing this task for all vertices, we end up with $c_{\ell}(v)$ being a vertex-colouring of $G$. A natural question to ask is whether $\ell$ can always be designed so that $c_{\ell}$ has particular properties.

For instance, one can require $c_{\ell}$ to be a proper colouring, i.e., to verify $c_{\ell}(u) \neq c_{\ell}(v)$ for every edge $u v$. This seems like a legitimate question, as proper colourings are perhaps the most investigated type of vertex-colourings. We say that a labelling $\ell$ is proper if $c_{\ell}$ is a proper colouring. A natural question is then: In general, what labels permit to design proper labellings? For a given graph $G$, we denote by $\chi_{\Sigma}(G)$ the least $k \geq 1$ (if any) such that $G$ admits proper $k$-labellings (i.e., labellings assigning labels from $\{1, \ldots, k\})$. Through inductive arguments, it is not complicated to prove that $\chi_{\Sigma}(G)$ is defined for every connected graph $G$ different from $K_{2}$; thus, in this context, we say that $G$ is nice whenever it has no component being $K_{2}$. The leading conjecture regarding the parameter $\chi_{\Sigma}$ is the well-known 1-2-3 Conjecture, raised in 2004 by Karoński, Łuczak and Thomason 9 .

Conjecture 1.1 (1-2-3 Conjecture [9]). For every nice graph $G$, we have $\chi_{\Sigma}(G) \leq 3$.

Many results have been obtained towards the 1-2-3 Conjecture; see [14] for a survey on this topic. The best result we have to date is that $\chi_{\Sigma}(G) \leq 5$ holds for every nice graph $G$ (see [8]). Let us also mention that determining whether $\chi_{\Sigma}(G) \leq 2$ holds for a given graph $G$ is NP-hard in general [6], but can be done in polynomial time when $G$ is bipartite [16].

* The first author was supported by a funding granted by the program "Jeunes Talents FRANCE-CHINE". The second author was supported by the National Natural Science Foundation of China (No. 11701440, 11626181). The third author was supported by the National Natural Science Foundation of China (No. 11601429) and the Fundamental Research Funds for the Central Universities (No. 3102019ghjd003). 
There are several ways for interpreting the 1-2-3 Conjecture. On the one hand, the conjecture states that for almost all graphs $G$, we should be able to "encode" a proper colouring via a labelling assigning labels with small value, no matter whether $\chi(G)$ (the chromatic number of $G$, i.e., the least number of colours in a proper colouring of $G$ ) is large or not. On the second hand, we note that, given a proper labelling $\ell$ of $G$, when replacing every edge $e=u v$ with $\ell(e)$ parallel edges joining $u$ and $v$, we end up with a multigraph $H$ which is locally irregular, i.e., for every edge $u v$ of $H$ we have $d(u) \neq d(v)$. So, in some sense, the 1-2-3 Conjecture states that nearly every graph $G$ can be turned into a locally irregular multigraph $H$ with the same structure (i.e., two vertices are adjacent in $H$ if and only if they are adjacent in $G$ ) by just replacing every edge with at most three parallel edges. As noted in [3], such concerns take place in a more general context where one aims at defining what an irregular graph should be, where the notion of local irregularity can then be perceived as an antonym of the notion of regularity.

In this work, we investigate how labellings can be used to generate other kinds of vertexcolourings, namely injective colourings. For a graph $G$, an injective colouring is a vertexcolouring where, for every vertex $v$, no two neighbours of $v$ get the same colour. In other words, every two distinct vertices are required to receive distinct colours as soon as there is a path of length 2 joining them. Equivalently, an injective colouring of $G$ can be seen as a proper colouring of $G^{(2)}$, the graph of the common neighbours of $G$ (i.e., $V\left(G^{(2)}\right)=V(G)$ and there is an edge joining $u$ and $v$ in $G^{(2)}$ if and only if $u$ and $v$ have a common neighbour in $G$ ). The least number of colours in an injective colouring of $G$ is denoted by $\chi_{\mathrm{i}}(G)$, which is called the injective chromatic number of $G$.

Injective colourings were first introduced in [7, where the authors raised several fundamental properties of these colourings. In particular, greedy colouring arguments show that $\chi_{\mathrm{i}}(G) \leq$ $\Delta(G)(\Delta(G)-1)+1$ holds for every graph $G$, while there do exist graphs for which the injective chromatic number reaches the upper bound (these graphs being exactly the incidence graphs of projective planes). Also, we clearly always have $\Delta(G) \leq \chi_{\mathrm{i}}(G)$ by the very definition of injective colouring. The authors of [7] also established that deciding whether $\chi_{\mathrm{i}}(G) \leq k$ holds for a given graph $G$ is NP-hard for every $k \geq 3$. Several other results on the topic appeared later in the literature, establishing mainly refined bounds for families of sparse graphs. We refer the interested reader to e.g. the pointers given in [11] for more details.

We call a labelling $\ell$ of a graph $G$ injective if $c_{\ell}$ is an injective colouring. We denote by $\chi_{\mathrm{i} \Sigma}(G)$ the least $k$ such that $G$ admits injective $k$-labellings. As will be shown in later Section 3 , let us mention that, this time, $\chi_{\mathrm{i} \Sigma}(G)$ is defined for every graph $G$. Studying injective labellings is motivated by the reasons exposed earlier. In particular, we note that, given an injective labelling $\ell$ of a graph $G$, when replacing the edges of $G$ by parallel edges as explained earlier, we here get a multigraph $H$ that is highly irregular (i.e., in which no vertex has two neighbours with the same degree), which is another possible antonym to regularity that was considered in [1].

Similarly as for the parameter $\chi_{\Sigma}$, our main concern is about how large can $\chi_{\mathrm{i} \Sigma}$ be in general. It is easy to see that, contrarily to the parameter $\chi_{\Sigma}(G)$, there is no absolute constant upper bound on $\chi_{\mathrm{i} \Sigma}(G)$ for every graph $G$, which can be as large as $\Delta(G)$ (any star is an example). As will be noted in upcoming Section 2, actually for every odd cycle $G$ we even have $\chi_{\mathrm{i} \Sigma}(G)=\Delta(G)+1$. Odd cycles are however the only such graphs we came up with, and, though it might seem daring, we would like to raise the following conjecture, which is our leading thread throughout this work.

Conjecture 1.2. For every graph $G$, we have $\chi_{\mathrm{i} \Sigma}(G) \leq \Delta(G)+1$. Furthermore, the upper bound is attained only when $G$ is an odd cycle.

This work is organised as follows. We start, in Section 2, by raising first observations on injective labellings, and showing that Conjecture 1.2 is true for some easy graph classes. In Section 3 , we establish bounds on $\chi_{\mathrm{i} \Sigma}(G)$ in terms of $\chi_{\mathrm{i}}(G)$. In particular, our bounds show that Conjecture 1.2 holds for some graphs $G$ verifying $\chi_{\mathrm{i}}(G)=\Delta(G)$. We then verify Conjecture 1.2 for more classes of graphs in Section 4 (trees, cacti, and subcubic graphs $G$ with $\chi_{\mathrm{i}}(G)=3$ ). In Section 5 , we establish that determining $\chi_{\mathrm{i} \Sigma}(G)$ for a given bipartite graph $G$ is an NP-hard problem. Conclusions and perspectives are presented in Section 6 


\section{First observations and warm-up results}

We start off with the following observation on labellings in general, which will be useful for proving one result later in this work.

Observation 2.1. Let $G$ be a graph, and $\ell$ be a labelling of $G$. Then

$$
\sum_{e \in E(G)} 2 \ell(e)=\sum_{v \in V(G)} c_{\ell}(v)
$$

In particular, by any labelling $\ell$, the sum $\sum_{v \in V(G)} c_{\ell}(v)$ must be even.

Proof. This is because every edge label contributes to the colour of exactly two vertices.

In the rest of this section, we provide some easy warm-up results towards Conjecture 1.2. First of all, we note that, in a complete graph $K_{n}$ with $n \geq 3$ vertices, every two vertices have common neighbours. Thus $\chi\left(K_{n}\right)=\chi_{\mathrm{i}}\left(K_{n}\right)$. By an injective labelling of $K_{n}$, we must thus make sure that all vertices get different colours. In other words, an injective labelling of $K_{n}$ is a proper labelling. Since complete graphs $K_{n}$ with $n \geq 3$ verify the 1-2-3 Conjecture [5], the following result holds, which shows that $\chi_{\mathrm{i} \Sigma}(G)$ can be much lower than $\Delta(G)$ in general.

Theorem 2.2. For every $n \geq 3$, we have $\chi_{\mathrm{i} \Sigma}\left(K_{n}\right)=3$.

We now consider complete bipartite graphs $K_{n, m}$, which also easily verify Conjecture 1.2 .

Theorem 2.3. For every $n, m \geq 1$ with $n \leq m$, we have $\chi_{\mathrm{i} \Sigma}\left(K_{n, m}\right) \leq m=\Delta\left(K_{n, m}\right)$.

Proof. Let $(U, V)$ denote the bipartition of $K_{n, m}$, where $U=\left\{u_{0}, \ldots, u_{n-1}\right\}$ and $V=\left\{v_{0}, \ldots, v_{m-1}\right\}$. Consider the following $m$-labelling $\ell$ of $K_{n, m}$. We first consider $v_{0} u_{0}$ and assign it label 1 . We then consider $v_{1} u_{1}$ and assign it label 2 . We go on like this for every $i \leq n-1$, and assign label $i+1$ to $v_{i} u_{i}$. For $v_{n}$ (if any), we "go back" to $u_{0}$ and assign label $n+1$ to $v_{n} u_{0}$. For $v_{n+1}$ (if any), we assign label $n+2$ to $v_{n+1} u_{1}$. And so on: for every vertex $v_{i}$ with $i \in\{n, \ldots, m-1\}$, we assign label $i+1$ to $v_{i} u_{i \bmod n}$. Finally, we assign label $m$ to all remaining edges of $K_{n, m}$.

Clearly, the maximum label value assigned by $\ell$ is exactly $m$. We claim that $\ell$ is injective. First of all, since $K_{n, m}$ is bipartite and complete, we only need to guarantee that all $u_{i}$ 's get different colours by $c_{\ell}$, and similarly for all $v_{i}$ 's. By construction of $\ell$, we note that for every vertex $v_{i}$, we have $c_{\ell}\left(v_{i}\right) \equiv i+1 \bmod m$. Thus, no two $v_{i}$ 's get the same colour. Now we note that due to the labelling scheme, we have $c_{\ell}\left(u_{j}\right)>c_{\ell}\left(u_{i}\right)$ whenever $j>i$. This is because, by how the procedure goes, labels $1, \ldots, m-1$ are assigned only once in such a way that whenever one of these labels is assigned to an edge incident to some $u_{i}$, then a strictly larger larger is assigned to an edge incident to every $u_{j}$ with $j>i$. Thus also no two $u_{i}$ 's can have the same colour, and $\ell$ is indeed injective.

In the next result, we prove Conjecture 1.2 for paths.

Theorem 2.4. For every path $G$, we have $\chi_{\mathrm{i} \Sigma}(G)=\Delta(G) \leq 2$.

Proof. If $G$ has length 1, then assigning label 1 to its unique edge results in an injective 1-labelling of $G$. So let us now focus on the general case. Let us denote by $v_{1}, \ldots, v_{n}$ the consecutive vertices of $G$, where $n \geq 3$. Since $n \geq 3$, note that $\chi_{\mathrm{i} \Sigma}(G)>1$. Consider the 2-labelling $\ell$ assigning labels $1,1,1,2,2,2,1,1,1,2,2,2, \ldots$ to the consecutive edges of $G$, from one end-edge to the other. Note that $\ell$ is injective (essentially because every two edges $v_{i} v_{i+1}, v_{i+3} v_{i+4}$ at distance 4 get different labels, and a situation where $v_{i+1} v_{i+2}$ gets the same label as $v_{i+3} v_{i+4}$ while $v_{i+2} v_{i+3}$ gets the same label as $v_{i} v_{i+1}$ never occurs; this leads the consecutive colours to be $1,2,2,3,4,4,3,2,2,3,4,4,3, \ldots)$, except in two cases:

- When the length of $G$ is 2 , in which case $c_{\ell}\left(v_{1}\right)=1=c_{\ell}\left(v_{3}\right)$. In that case, assigning labels 1,2 to the edges yields an injective labelling.

- When the length of $G$ is congruent to 4 modulo 6 , in which case we get $c_{\ell}\left(v_{n}\right)=2=c_{\ell}\left(v_{n-2}\right)$. Note however that this conflict is unique. Here, assigning $1,1,2,2,2,1,1,1,2,2,2,1,1,1, \ldots$. to the consecutive edges of $G$ instead results in an injective 2-labelling. 
We now prove Conjecture 1.2 for cycles. We start off by considering even cycles, i.e., cycles with even length.

Theorem 2.5. For every even cycle $G$, we have $\chi_{\mathrm{i} \Sigma}(G)=2=\Delta(G)$.

Proof. We denote by $v_{0}, \ldots, v_{n-1}$ the consecutive vertices of $G$, where $n$ is even. Obviously, we have $\chi_{\mathrm{i} \Sigma}(G)>1$. Let us show that $\chi_{\mathrm{i} \Sigma}(G)=2$.

- First assume $G$ has length $4 k$. We produce a 2-labelling $\ell$ where, as going along the consecutive vertices, the colours by $c_{\ell}$ are $3,3,2,2,3,3,2,2,3, \ldots$ Note that any labelling with this property is indeed injective. To get $\ell$, we consider the set of edges $F=\left\{v_{i} v_{i+1}: i \equiv 0 \bmod 4\right\}$ of $G$, assign label 2 to all edges of $F$, and assign label 1 to all remaining edges. It is then easy to see that, due to the value of $n$, the colouring $c_{\ell}$ assigns the desired colours (we have $c_{\ell}\left(v_{i}\right)=3$ if $i \equiv 0,1 \bmod 4$, and $c_{\ell}\left(v_{i}\right)=2$ otherwise). Note that by turning all 1 's by $\ell$ into 2 's and vice versa, we would as well obtain an injective 2-labelling where the resulting vertex colours alternate between pairs of 2's and pairs of 4's.

- Now assume $G$ has length $4 k+2$. Let $G^{\prime}$ be the cycle obtained from $G$ by contracting the edges $v_{1} v_{2}$ and $v_{2} v_{3}$. Note that $G^{\prime}$ has length $4 k$. Thus, it admits an injective 2-labelling $\ell^{\prime}$. Actually, applying the arguments we used in the previous case above, we can assume that $v_{0}$ and $v_{3}$ (resulting from the contractions) have colour $3, v_{n-1}$ and $v_{4}$ have colour 2 , and the edge $v_{0} v_{3}$ in $G^{\prime}$ is labelled 2 (which implies that both $v_{n-1} v_{0}$ and $v_{3} v_{4}$ are labelled 1 ). We extend $\ell^{\prime}$ to a 2-labelling $\ell$ of $G$ by just, in $G$, assigning label 2 to all of $v_{0} v_{1}, v_{1} v_{2}$ and $v_{2} v_{3}$. This way, every vertex $v_{i}$ in $V(G) \backslash\left\{v_{1}, v_{2}\right\}$ verifies $c_{\ell}\left(v_{i}\right)=c_{\ell^{\prime}}\left(v_{i}\right)$. Furthermore, we have $c_{\ell}\left(v_{1}\right)=c_{\ell}\left(v_{2}\right)=4$, and only these two vertices have colour 4 . Then it is easy to see that $\ell$ is an injective 2-labelling of $G$.

Let us now consider odd cycles, i.e., cycles with odd length.

Theorem 2.6. For every odd cycle $G$, we have $\chi_{\mathrm{i} \Sigma}(G)=3=\Delta(G)+1$.

Proof. Let us first prove that $\chi_{\mathrm{i} \Sigma}(G)>2$. Suppose this is wrong, and let $G$ be an odd cycle admitting an injective 2-labelling $\ell$. Since all vertices of $G$ have degree 2, their possible colours by $\ell$ are 2, 3 and 4. Furthermore, for a vertex to have colour 2, its two incident edges must be labelled 1, while, for a vertex to have colour 4, its two incident edges must be labelled 2 . This means that $G$ has no edge $u v$ such that $c_{\ell}(u)=2$ and $c_{\ell}(v)=4$. So a vertex with colour 2 must neighbour vertices with colour 2 or 3 , and a vertex with colour 4 must neighbour vertices with colour 4 or 3 . Since no vertex can have its two neighbours having the same colour by $c_{\ell}$, this means that the vertices of $G$ with colour 2 induce a matching, and similarly for the vertices with colour 4 . If we denote by $n_{i}$ the number of vertices with colour $i$, then we have that $n_{2}$ and $n_{4}$ are even, while $n_{3}$ must be odd since $n_{1}+n_{2}+n_{3}=|V(G)|$ is odd. We then get a contradiction, because the sum of the colours by $c_{\ell}$, which is $2 n_{2}+3 n_{3}+4 n_{4}$, is odd, which is impossible by Observation 2.1.

Let us now prove that $\chi_{\mathrm{i} \Sigma}(G)=3$. We denote by $v_{0}, \ldots, v_{n-1}$ the consecutive vertices of $G$, where $n$ is odd. If $n=3$, then it is easy to see that we must assign different labels to all edges, and the claim holds. Now consider $G$ a general odd cycle with $n \geq 5$ vertices. Let $G^{\prime}$ be the cycle obtained by contracting the edge $v_{1} v_{2}$; note that $G^{\prime}$ has length $n-1$. By Theorem 2.5, there is then an injective 2-labelling $\ell^{\prime}$ of $G^{\prime}$ which we would like to extend to an injective 3-labelling $\ell$ of $G$. Since $\ell^{\prime}$ cannot assign label 1 only, we may suppose that $\ell^{\prime}\left(v_{0} v_{2}\right)=2$ (calling $v_{2}$ the vertex resulting from the contraction). Also, as can be checked from the proof of Theorem 2.5, we may assume that $c_{\ell^{\prime}}\left(v_{0}\right)=c_{\ell^{\prime}}\left(v_{2}\right)=4$ (either $G^{\prime}$ has length $4 k$ and we can flip labels as explained earlier, or $G^{\prime}$ has length $4 k+2$ in which case a pair of adjacent vertices with colour 4 is created in the proof above). We extend $\ell^{\prime}$ to $G$ by assigning label 2 to $v_{0} v_{1}$ and label 3 to $v_{1} v_{2}$. This way we get $c_{\ell}\left(v_{0}\right)=4, c_{\ell}\left(v_{1}\right)=5$ and $c_{\ell}\left(v_{2}\right)=5$. Since $c_{\ell}\left(v_{0}\right)=c_{\ell^{\prime}}\left(v_{0}\right)$ and all vertices different from $v_{1}$ and $v_{2}$ have the same colour by $\ell^{\prime}$ and $\ell$ (which is at most 4 ), no conflict arises between $v_{0}$ and another vertex. Similarly, $v_{1}$ and $v_{2}$ are the only two vertices with colour 5 , and they do not share any neighbour since $n \geq 5$. Thus, $\ell$ is an injective 3-labelling of $G$. 


\section{Bounding $\chi_{\mathrm{i} \Sigma}(G)$ above by a function of $\chi_{\mathrm{i}}(G)$}

We here show how injective colourings can help to design injective labellings. Towards Conjecture 1.2 , this is particularly useful for graphs $G$ where $\chi_{\mathrm{i}}(G)$ is close to $\Delta(G)$.

\subsection{On switching odd and even walks}

Our proofs will repeatedly modify labels along walks with certain length, that are well known to exist under certain circumstances. This approach is actually a rather common one for designing distinguishing labellings, see e.g. 2, 4, 5, 9, 10, 15, 16. Recall that for two (not necessarily different) vertices $u, v$ of a graph, a $(u, v)$-walk (or walk, for short) is a path from $u$ to $v$ with possible vertex and edge repetitions. Let us emphasize that a $(u, v)$-walk is not the same as a $(v, u)$-walk; in our proofs below, it is actually important which vertex is the starting point of the walk, and which vertex is the ending point. A $(u, u)$-walk is called a closed walk. A walk is said even if its length is even, while it is said odd otherwise.

Lemma 3.1. Let $G$ be a connected non-bipartite graph, and $u$ and $v$ be two (not necessarily distinct) vertices of $G$. Then $G$ has both even $(u, v)$-walks and odd $(u, v)$-walks.

Proof. Since $G$ is not bipartite, it has an odd cycle $C$. Then consider, in $G$, a walk $P$ from $u$ to a vertex $w$ of $C$, and a walk $P^{\prime}$ from $w$ to $v$. Possibly, $w \in\{u, v\}$. Then $\left(u, P, w, P^{\prime}, v\right)$ and $\left(u, P, w, C, w, P^{\prime}, v\right)$ are two $(u, v)$-walks of $G$ with different length parity.

Lemma 3.2. Let $G$ be a connected bipartite graph, and $u$ and $v$ be two (not necessarily distinct) vertices of $G$. Then:

- if $u$ and $v$ belong to different partite sets, then all $(u, v)$-walks are odd;

- otherwise, i.e., $u$ and $v$ belong to the same partite set, then all $(u, v)$-walks are even.

Proof. This follows trivially from the bipartition of $G$.

When designing labellings, a common approach is by repeatedly considering pairs of vertices $u, v$ and switching labels along the edges of a $(u, v)$-walk $P$. Let $\ell$ be a $\{0, \ldots, k-1\}$-labelling of a graph $G$. For some number $\alpha$, by $\alpha$-switching $P$ we mean modifying the labels assigned to the edges of $P$, traversing it from $u$ to $v$, in the following way: we apply $+\alpha$ to the label of the first edge, $-\alpha$ to the label of the second edge, $+\alpha$ to the label of the third edge, $-\alpha$ to the label of the fourth edge, and so forth, where the operations are understood modulo $k$. This switching operation has the following properties:

Observation 3.3. Let $G$ be a graph and $\ell$ be $a\{0, \ldots, k-1\}$-labelling of $G$. Let $P$ be $a(u, v)$-walk of $G$, and let $\ell^{\prime}$ be the labelling of $G$ obtained from $\ell$ by $\alpha$-switching $P$ for some $\alpha$. Then:

- for every inner vertex $w$ of $P$, we have $c_{\ell^{\prime}}(w) \equiv c_{\ell}(w) \bmod k$;

- if $P$ is even, then $c_{\ell^{\prime}}(u) \equiv c_{\ell}(u)+\alpha \bmod k$ and $c_{\ell^{\prime}}(v) \equiv c_{\ell}(v)-\alpha \bmod k$;

- if $P$ is odd, then $c_{\ell^{\prime}}(u) \equiv c_{\ell}(u)+\alpha \bmod k$ and $c_{\ell^{\prime}}(v) \equiv c_{\ell}(v)+\alpha \bmod k$.

Proof. The first item is because for every inner vertex $w$ of $P$, we have $c_{\ell^{\prime}}(w)=c_{\ell}(w)+\alpha-\alpha$. The two last items are deduced from the length of $P$, and the fact that, when $\alpha$-switching, we alternate between additions and subtractions (by $\alpha$ ) as going from $u$ to $v$.

In the next series of results, we show how an initial vertex-colouring of a graph can serve as a layout for designing labellings with specific colouring properties.

Lemma 3.4. Let $G$ be a connected non-bipartite graph, and $\left(V_{0}, \ldots, V_{k-1}\right)$ be a vertex-colouring (with no specific properties) of $G$ with $k \not \equiv 2 \bmod 4$. Then $G$ admits a $k$-labelling $\ell$ such that, for every $i \in\{0, \ldots, k-1\}$ and every vertex $v \in V_{i}$, we have $c_{\ell}(v) \equiv i \bmod k$.

Proof. Aiming at colours modulo $k$, note that we can equivalently look for $\ell$ being a $\{0, \ldots, k-1\}$ labelling (since labels 0 and $k$ are equivalent modulo $k$ ). We distinguish a few cases: 
- Assume an even number $x \geq 0$ of the $V_{i}$ 's are odd (i.e., of odd cardinality), and that at least $y \geq 1$ of the $V_{i}$ 's are even (i.e., of even cardinality). Free to relabel the indexes, we can assume that $V_{0}, V_{\frac{x}{2}+1}, \ldots, V_{k-\frac{x}{2}-1}$ are even while $V_{1}, \ldots, V_{\frac{x}{2}}, V_{k-\frac{x}{2}}, \ldots, V_{k-1}$ are odd. Note that this relabelling is correct (i.e., every colour class is relabelled, and no two colour classes get relabelled the same way) due to our assumption on $k$. We start from $\ell$ assigning 0 to all edges of $G$. Note that all vertices of $V_{0}$ are then good, i.e., for every $v \in V_{0}$ we have $c_{\ell}(v) \equiv 0 \bmod k$, while every other vertex is bad, i.e., for every $v \in V_{i}$ with $i \neq 0$ we have $c_{\ell}(v) \not \equiv i \bmod k$. Our goal is to make all these bad vertices good, and, for that, we modify $\ell$ by $\alpha$-switching some walks joining bad vertices.

Consider two bad vertices $u$ and $v$ of $V_{\frac{x}{2}+1}$ (if any; however, due to its cardinality, if this set is not empty, then it has at least two vertices). Let $P$ be an odd $(u, v)$-walk of $G$; such exists by Lemma 3.1 since $G$ is not bipartite. Now $\left(\frac{x}{2}+1\right)$-switch $P$; by Observation 3.3 all bad vertices different from $u$ and $v$ remain bad (with colour 0 modulo $k$ ), while the colour of $u$ and $v$ becomes $\frac{x}{2}+1$ modulo $k$. Thus, $u$ and $v$ become good and all other vertices remain bad. By repeating this argument for pairs of bad vertices of $V_{\frac{x}{2}+1}, \ldots, V_{k-\frac{x}{2}-1}$ (where, for two vertices of $V_{i}$, odd walks should be $i$-switched), we can make all their vertices good. Recall in particular that all those $V_{i}$ 's have an even number of vertices.

Quite similarly, by switching odd walks joining vertices of $V_{1}, \ldots, V_{\frac{x}{2}}, V_{k-\frac{x}{2}}, \ldots, V_{k-1}$, we can make sure that the only remaining bad vertices are $v_{1}, \ldots, v_{\frac{x}{2}}, v_{k-\frac{x}{2}}, \ldots, v_{k-1}$, where $v_{i} \in V_{i}$ for every $i \in\left\{1, \ldots, \frac{x}{2}, k-\frac{x}{2}, \ldots, k-1\right\}$. That is, there remain $x$ bad vertices, one in each of $V_{1}, \ldots, V_{\frac{x}{2}}, V_{k-\frac{x}{2}}, \ldots, V_{k-1}$. We make them good in pairs. To achieve this, we consider each two bad $v_{i}$ and $v_{k-i}$, an even $\left(v_{i}, v_{k-i}\right)$-walk $P$ joining them, and we $i$-switch $P$. This way, by Observation 3.3 the colour of $v_{i}$ is altered by $i$ modulo $k$ (which then becomes $i$ modulo $k$ ), while the colour of $v_{k-i}$ is altered by $-i$ modulo $k$ (which then becomes $k-i$ modulo $k$ ). Also, all other bad vertices remain of colour 0 modulo $k$. Once every pair of remaining bad vertices has been considered, we then end up with the desired $\ell$.

- Assume an odd number $x \geq 1$ of the $V_{i}$ 's are odd, and there are $y \geq 0$ even $V_{i}$ 's. In that case, we relabel the indexes of the $V_{i}$ 's so that $V_{0}, V_{1}, \ldots, V_{\frac{x-1}{2}}, V_{k-\frac{x-1}{2}}, \ldots, V_{k-1}$ are odd, while $V_{\frac{x-1}{2}+1}, \ldots, V_{k-\frac{x-1}{2}-1}$ are even. Again, this relabelling is correct. As in the previous case, we start from $\ell$ assigning 0 to all edges of $G$ so that all vertices of $V_{0}$ are good. Now, note that, omitting $V_{0}$, the number of odd $V_{i}$ 's is even. Quite similarly as in the previous case, we can then make all vertices good, by first making good pairs of vertices from the even $V_{i}$ 's, and then making good pairs of vertices from the remaining even number of odd $V_{i}$ 's.

- The last case to consider is when all $V_{i}$ 's are odd, and there are an even number of them. Recall that $k \not \equiv 2 \bmod 4$; thus $k \equiv 0 \bmod 4$. This means that $\frac{k}{2}$ is even. In that case, we proceed as follows. We start from $\ell$ assigning label 0 to all edges, so that all vertices of $V_{0}$ are good. Just as in the previous cases, we then switch weights along odd walks until we get to the point when the remaining bad vertices are $v_{1}, \ldots, v_{k-1}$, where $v_{i} \in V_{i}$ for every $i \in\{1, \ldots, k-1\}$. As in the first case above, by then 1 -switching an odd $\left(v_{1}, v_{k-1}\right)$-walk, then 2-switching a $\left(v_{2}, v_{k-2}\right)$-walk, and so on, we get to the point where, due to the value of $k$, only $v_{\frac{k}{2}}$ is bad. Recall that $v_{\frac{k}{2}}$ has colour 0 modulo $k$, and $\frac{k}{2}$ is even. We here consider an odd $\left(v_{\frac{k}{2}}, v_{\frac{k}{2}}\right)$-walk containing $v_{\frac{k}{2}}$, which we $\frac{k}{4}$-switch. By Observation 3.3 this alters the colour of $v_{\frac{k}{2}}$ by $\frac{k}{2}$, which then becomes good.

When $G$ is not bipartite and the provided vertex-colouring $\left(V_{0}, \ldots, V_{k-1}\right)$ verifies $k \equiv 2 \bmod 4$, there are cases where, depending on the parity of the $V_{i}$ 's, the same conclusion can be reached.

Lemma 3.5. Let $G$ be a connected non-bipartite graph, and $\left(V_{0}, \ldots, V_{k-1}\right)$ be a vertex-colouring (with no specific properties) of $G$ with $k \equiv 2 \bmod 4$. If not all $V_{i}$ 's are odd, then $G$ admits a $k$ labelling $\ell$ such that, for every $i \in\{0, \ldots, k-1\}$ and every vertex $v \in V_{i}$, we have $c_{\ell}(v) \equiv i \bmod k$.

Proof. If some of the $V_{i}$ 's are even, then we note that some arguments used in the proof of Lemma 3.4 apply the same way, and we can deduce $\ell$ in a similar manner. 
For the proof of Lemma 3.4 to work, it is important that the layout vertex-colouring has convenient parity properties, and that the graph has odd walks joining any pair of vertices. The latter point is why the situation is a bit more troublesome for bipartite graphs. However, we note that the switching operation can be employed to get a result close to Lemma 3.4 for any graph.

Lemma 3.6. Let $G$ be a connected graph, and $\left(V_{0}, \ldots, V_{k-1}\right)$ be a vertex-colouring (with no specific properties) of $G$. Let $v^{*}$ be any vertex of $G$, where $v^{*} \in V_{x}$ for some $x \in\{0, \ldots, k-1\}$. Then $G$ admits a $k$-labelling $\ell$ such that, for every $i \in\{0, \ldots, k-1\}$ and every vertex $v \in V_{i}$ different from $v^{*}$, we have $c_{\ell}(v) \equiv i \bmod k$.

Proof. Again, we can equivalently assume that $\ell$ assigns labels in $\{0, \ldots, k-1\}$. Start from $\ell$ assigning label 0 to all edges. Then repeatedly consider a vertex $v \neq v^{*}$, consider any $\left(v, v^{*}\right)$-walk $P$, and, assuming $v \in V_{i}$, just $i$-switch $P$. By Observation 3.3, this makes $v$ good, and all vertices different from $v^{*}$ that have not been treated yet remain bad with colour 0 modulo $k$. Once all vertices have been treated this way, the only remaining bad vertex is $v^{*}$.

\subsection{Upper bounds on $\chi_{\mathrm{i \Sigma}}(G)$}

We now show how to apply the previous results to deduce upper bounds on $\chi_{\mathrm{i} \Sigma}(G)$ being functions of $\chi_{\mathrm{i}}(G)$. We start off with the nicest case.

Theorem 3.7. Let $G$ be a connected non-bipartite graph with $\chi_{\mathrm{i}}(G) \not \equiv 2 \bmod 4$. Then, $\chi_{\mathrm{i} \Sigma}(G) \leq$ $\chi_{\mathrm{i}}(G)$.

Proof. Let $\left(V_{0}, \ldots, V_{k-1}\right)$ be an injective $k$-colouring of $G$, where $k=\chi_{\mathrm{i}}(G)$. Applying Lemma 3.4 on that vertex-colouring, we get that there exists a $k$-labelling $\ell$ of $G$ where, for every vertex $v \in V_{i}$, we have $c_{\ell}(v) \equiv i \bmod k$. Since $\left(V_{0}, \ldots, V_{k-1}\right)$ is injective, it is easy to see that $\ell$ is as well.

We now deal with the remaining two cases. That is, we establish an upper bound, function of $\chi_{\mathrm{i}}(G)$, on $\chi_{\mathrm{i} \Sigma}(G)$ whenever $G$ is not bipartite and $\chi_{\mathrm{i}}(G) \equiv 2 \bmod 4$, and when $G$ is bipartite.

Theorem 3.8. Let $G$ be a connected non-bipartite graph with $\chi_{\mathrm{i}}(G) \equiv 2 \bmod 4$. Then, $\chi_{\mathrm{i} \Sigma}(G) \leq$ $\chi_{\mathrm{i}}(G)+1$.

Proof. Let $\left(V_{0}, \ldots, V_{k-1}\right)$ be an injective $k$-colouring of $G$, where $k=\chi_{\mathrm{i}}(G)$. Recall that $k \equiv$ $2 \bmod 4$. If some of the $V_{i}$ 's are even, then Lemma 3.5 applies. So the remaining case is when all $V_{i}$ 's are odd; in that case, we note that none of the labelling schemes described in Lemma 3.4 applies (in particular, because $\frac{k}{2}$ is not even). In that situation, we relabel the colour classes as $\left(V_{1}, \ldots, V_{k}\right)$, and we aim at designing an injective $\{0, \ldots, k\}$-labelling $\ell$ where, for every vertex $v$ lying in part $V_{i}$, we have $c_{\ell}(v) \equiv i \bmod k+1$.

Quite similarly as in the proof of Lemma 3.4. by switching odd walks we can reach a situation where all vertices but $v_{1}, \ldots, v_{k}$ are good, where $v_{i} \in V_{i}$ for every $i \in\{1, \ldots, k\}$. To make these last $k$ vertices good, we switch even walks as follows. We first consider an even $\left(v_{1}, v_{k}\right)$-walk of $G$ (which exists by Lemma 3.1), which we 1-switch. By Observation 3.3 this makes both $v_{1}$ and $v_{k}$ good (modulo $k+1)$. We then consider an even $\left(v_{2}, v_{k-1}\right)$-walk which we 2 -switch, thereby making $v_{2}$ and $v_{k-1}$ good. We go on that way, considering an even $\left(v_{i}, v_{k-i+1}\right)$-walk which we $i$-switch, for every $i \in\left\{1, \ldots, \frac{k}{2}\right\}$. Note that this is well defined since $k$ is even. This eventually makes all $v_{i}$ 's good, and thus $\ell$ an injective $\{0, \ldots, k\}$-labelling of $G$.

Theorem 3.9. For every bipartite graph $G$, we have $\chi_{\mathrm{i} \Sigma}(G) \leq \chi_{\mathrm{i}}(G)+1$.

Proof. We may assume that $G$ is connected. Let $(U, V)$ denote the bipartition of $G$. Let $\left(V_{0}, \ldots, V_{k}\right)$ be an injective colouring of $G$ with $V_{k}$ empty, where $k=\chi_{\mathrm{i}}(G)$. We aim at designing an injective $\{0, \ldots, k\}$-labelling $\ell$ of $G$ where, for most vertices $v$, we have $c_{\ell}(v) \equiv i \bmod k+1$ (where $\left.v \in V_{i}\right)$. An important thing to note is that only vertices in the same partite set of $G$ can be joined by a path of length 2. This means that we can focus our attention on making sure that the vertices in $U$ have a desired colour by $c_{\ell}$, and independently do the same with the vertices in $V$. The problem here is that, unlike in the non-bipartite case, we do not have odd walks joining vertices in a same partite set. 
Since only vertices from a same partite set can be joined by a path of length 2 , either $\mid \bigcup_{i=0}^{k} U \cap$ $V_{i} \mid \geq k$ or $\left|\bigcup_{i=0}^{k} V \cap V_{i}\right| \geq k$. Let us thus assume that $U$ contains at least one vertex from each $V_{i}$, i.e., $|U| \geq k$. Using Lemma 3.6, we can reach a $\{0, \ldots, k\}$-labelling $\ell$ where, for every vertex $v \in V_{i}$ but some $v^{*}$ in $U \cap V_{0}$, we have $c_{\ell}(v) \equiv i \bmod k+1$. If $c_{\ell}\left(v^{*}\right) \equiv 0 \bmod k+1$ or $c_{\ell}\left(v^{*}\right) \equiv k \bmod k+1$, then we are done; so assume this is not the case. By the initial injective colouring, recall that there is no vertex in $V_{k}$, and thus no vertex $v$ with $c_{\ell}(v) \equiv k \bmod k+1$. To fix the colour of $v^{*}$, we proceed as follows. Consider a vertex $v \in V_{k-1} \cap U$, and 1-switch a $\left(v, v^{*}\right)$-walk. This makes the colour of $v^{*}$ decrease by 1 (modulo $k+1$ ), while now $c_{\ell}(v) \equiv k \bmod k+1$, which raises no conflict since no other vertex has this property. If now $c_{\ell}\left(v^{*}\right) \equiv 0 \bmod k+1$, then we are done. Otherwise, we repeatedly consider another vertex $v \in U \cap V_{k-1}$ (if any) and 1-switch a $\left(v, v^{*}\right)$-path, until hopefully $v^{*}$ gets colour 0 modulo $k+1$. Note that vertices that originally were in $V_{k-1}$ raise no conflict as long as their colour is $k-1$ or $k$ modulo $k+1$, since no two of them are joined by a path of length 2 .

If we reach the point where there is no more vertex $v$ with $c_{\ell}(v) \equiv k-1 \bmod k+1$, but $v^{*}$ still does not have colour 0 modulo $k+1$, then we repeat this process with the vertices having colour $k-2$. That is, at this point no vertex has colour $k-1$ modulo $k+1$. So we can again freely consider vertices $v \in V \in U \cap V_{k-2}$, and 1-switch a $\left(v, v^{*}\right)$-walk to decrease the colour of $v^{*}$ by 1 , while making the colour of $v$ being $k-1$ modulo $k+1$. If at some point $v^{*}$ gets a desired colour, then we are done. Otherwise, we get to the point where no more vertex has colour $k-2$ modulo $k+1$, and we can then consider the vertices with colour $k-3$, and so on. Since $|U| \geq k$, by repeating this process we can make $v^{*}$ reach the desired colour, so that all vertices that originally were in a same $V_{i}$ have the same colour $(i$ or $i+1)$ modulo $k+1$, except possibly for one class $V_{x}$ whose some vertices have colour $x$ modulo $k+1$ while the others have colour $x+1$ modulo $k+1$.

\section{Other classes of graphs verifying Conjecture 1.2}

In this section, we verify Conjecture 1.2 for a few more classes of graphs with injective chromatic number close to the maximum degree. We consider trees, cacti, and some subcubic graphs.

\subsection{Trees}

We start by verifying Conjecture 1.2 in the case of trees. Recall that trees $T$ verify $\chi_{\mathrm{i}}(T) \leq \Delta(T)$.

Observation 4.1. For every tree $T$, we have $\chi_{\mathrm{i}}(T) \leq \Delta(T)$

Proof. This can be proved by induction. The base case is that of a star $T$, in which case an injective $\Delta(T)$-colouring is obtained by assigning a distinct colour to all leaves, and any colour to the center. In the general case of a tree $T$, consider a leaf $v$ with unique neighbour $u$. An injective $\Delta(T)$-colouring of $T-v$ (obtained by induction) can then be extended to $v$ easily, since the only colours that cannot be assigned to $v$ are those assigned to the neighbours of $u$ in $T-v$. Since $u$ has at most $\Delta(T)-1$ neighbours in $T-v$, there is at least one open colour for $v$.

Regarding proving Conjecture 1.2 for trees, a surprising fact is that simple counting arguments fail to make a straight induction scheme work. Also, trees are bipartite, and this is one of those conditions where our results from Section 3 do not give the result we want immediately. The proof we give actually makes use of Lemma 3.6 .

Theorem 4.2. For every tree $T$, we have $\chi_{\mathrm{i} \Sigma}(T) \leq \Delta(T)$.

Proof. Set $\Delta=\Delta(T)$. If $T$ is a star, then the $\Delta$-labelling assigning a different label to every edge is clearly injective. So we can assume that $T$ is not a star. Let $T$ be rooted at any vertex $r$ with degree at least 3 , and consider a vertex $v \neq r$ whose all sons $u_{1}, \ldots, u_{d}$ are leaves (where $1 \leq d \leq \Delta-1)$. Let $w$ denote the parent of $v$. Recall that $w$ has degree at least 2 , since $T$ is not a star.

Let $T^{\prime}=T-\left\{u_{1}, \ldots, u_{d}\right\}$. Let $\left(V_{0}, \ldots, V_{\Delta-1}\right)$ be an injective $\Delta$-colouring of $T^{\prime}$, which exists by Observation 4.1. Free to relabel the indexes, we may assume that $w \in V_{1}$. Now, by Lemma 3.6. there is a labelling $\ell^{\prime}$ of $T^{\prime}$ where $v^{*}=v$ is potentially the only vertex that does not verify 
$c_{\ell^{\prime}}(v) \equiv x \bmod \Delta\left(\right.$ where $\left.v \in V_{x}\right)$. Since $w \in V_{1}$ and $d(w) \geq 2$, note that $c_{\ell^{\prime}}(w) \geq \Delta+1$. We want to extend $\ell^{\prime}$ to an injective $\Delta$-labelling $\ell$ of $T$, by correctly assigning a label to each of $v u_{1}, \ldots, v u_{d}$. When assigning a label $\alpha$ to $v u_{i}$ by $\ell$, the colour of $u_{i}$ becomes $\alpha$. Since the labels we assign are $1, \ldots, \Delta$, the colour of $u_{i}$ will necessarily be at most $\Delta$. Furthermore, the only vertices joined with $u_{i}$ via a path of length 2 are the other $u_{i}$ 's and $w$, while $w$ was shown to have colour at least $\Delta+1$. Thus, from the point of view of the $u_{i}$ 's, no conflict can arise as long as every two of the $v u_{i}$ 's are assigned distinct labels by $\ell$. Now, when labelling the $v u_{i}$ 's, we also affect the colour of $v$. However, by how $\ell^{\prime}$ was obtained, note that $v$ cannot be involved in a conflict as soon as its colour gets congruent to $x$ modulo $\Delta$.

Following these arguments, $\ell$ can be obtained from $\ell^{\prime}$ by assigning distinct labels to $v u_{1}, \ldots, v u_{d}$ so that the sum of the assigned labels is $x-c_{\ell^{\prime}}(v)$ modulo $\Delta$, which is possible to achieve since $1 \leq d \leq \Delta-1$ and we are assigning labels in $\{1, \ldots, \Delta\}$. To see this is true, consider for instance the following procedure. Start from each $v u_{i}$ being labelled $i$. Then repeatedly increment the label of $v u_{d}$ until its label becomes $\Delta$. So far, we have already generated $\Delta-d+1$ sums. Then, for each successive value of $i=d-1, \ldots, 1$, increment the label of $v u_{i}$ once. This generates $d-1$ more sums. In total, we have thus generated $\Delta$ sums, and at each step it can be noted that no two of the $v u_{i}$ 's are assigned a same label.

\subsection{Cacti}

We partially extend the previous result to the class of cacti, where, recall, a cactus is a graph in which every two cycles intersect on at most one vertex. We say that a cactus is even if it is bipartite, while it is odd otherwise.

Observation 4.3. For every cycle $G$, we have $\chi_{\mathrm{i}}(G) \leq 3$.

Proof. If $G=C_{2 k}$ is even, then we are done by applying colours $\alpha_{0}, \alpha_{0}, \alpha_{1}, \alpha_{1}, \alpha_{2}, \alpha_{2}, \ldots, \alpha_{k-1}, \alpha_{k-1}$ (where the $\alpha_{i}$ 's belong to $\{1,2,3\}$ ) to the consecutive vertices of $C$ in such a way that, modulo $k$, we have $\alpha_{i} \neq \alpha_{i+1}$ for every $i \in\{0, \ldots, k-1\}$. Such a pattern exists when three colours are used. If $G=C_{2 k+1}$ is odd, then we can basically consider such an injective 3-colouring for $C_{2 k+2}$ where $\alpha_{0} \neq \alpha_{2}$, and contract a vertex with colour $\alpha_{1}$ while keeping the colours of the other vertices. This yields an injective 3 -colouring of $G$.

Note that in cacti with maximum degree at least 3 as well, the injective chromatic number is very close to the lower bound.

Lemma 4.4. Let $G$ be a cactus with maximum degree $\Delta \geq 3$. Then:

- if $G$ is even, then $\chi_{\mathrm{i}}(G)=\Delta$;

- if $G$ is odd, then $\chi_{\mathrm{i}}(G) \in\{\Delta, \Delta+1\}$.

Furthermore, there exist odd cacti $G$ with maximum degree $\Delta \geq 3$ verifying $\chi_{\mathrm{i}}(G)=\Delta+1$.

Proof. We prove the claim for even cacti first. The proof is by induction. Since the claim can be checked by hand when $G$ is small, we focus on the general case. If $G$ has a leaf $v$ with unique neighbour $u$, then we note that an injective $\Delta$-colouring of $G-v$ (obtained by induction, or by Observation 4.3 can be extended to $v$, thus to $G$, since we have $\Delta$ colours in hand and we just need to assign to $v$ a colour different from the colours of the at most $\Delta-1$ other neighbours of $u$. Thus $G$ is just made of cycles joined by (possibly length-0) paths, every two of these cycles sharing at most one vertex. There is thus, in $G$, a cycle $C$ whose all vertices but one, say $u$, have degree 2 in $G$. This is what we call an end-cycle. Let $v$ be a vertex of $C$ which is as far as possible from $u$. Then $v$ has degree 2 with two neighbours $v_{1}, v_{2}$ of degree 2 .

We deduce an injective $\Delta$-colouring of $G-v$ by induction. Assume first $C$ has even length at least 6 . Since $v_{1}$ has degree 1 in $G-v$, its colour must only be different from the colour assigned to the unique other vertex adjacent to its unique neighbour. Thus, we can recolour $v_{1}$, if necessary, to make sure that $v_{1}$ and $v_{2}$ have different colours. This ensures that this colouring remains valid in $G$, and we are left with finding an open colour for $v$. By our choice of $v$, it can be checked that at most two colours are forbidden at $v$ since $|C| \geq 6$; here we are done since we are colouring 
the graph with at least three colours. When $C$ has length 4 , then we note that, by the injective $\Delta$-colouring of $G-v$, it already holds that $v_{1}$ and $v_{2}$ have different colours. Thus the colouring is valid in $G$ as well. Then the previous arguments apply just the same for finding an open colour for $v$.

We now focus on odd cacti. Let us proceed by induction again. We note that all arguments used in the previous case also apply here. The only difference here is that $|C|$ might be odd. If $|C| \geq 5$, then we note that the arguments used earlier when $|C| \geq 6$ apply here. So the remaining case is when $C$ is a triangle $\left(v, v_{1}, v_{2}, v\right)$. Here, we consider $G^{\prime}=G-\left\{v_{1}, v_{2}\right\}$. By induction, there is an injective $(\Delta+1)$-colouring of $G^{\prime}$. When extending this colouring to $v_{1}$ and $v_{2}$, we need to make sure that the assigned colours are different from the at most $\Delta-2$ other neighbours of $v$, and also from the colour of $v$ since $v, v_{1}$ and $v_{2}$ form a triangle. This argument also implies that $v_{1}$ and $v_{2}$ must be assigned different colours. Since we have $\Delta+1$ colours in hand, we can correctly extend the colouring to $v_{1}$ and $v_{2}$, thus to an injective $(\Delta+1)$-colouring of $G$.

Regarding the very last part of the statement, we note that a cactus $G$ with even maximum degree $\Delta$ verifies $\chi_{\mathrm{i}}(G)=\Delta+1$ as soon as $G$ has a fan vertex, which we define as a vertex with degree $\Delta$ to which are attached $\Delta / 2$ triangles. As will be remarked later, there are actually other types of structures that force the injective chromatic number of a cactus with maximum degree $\Delta$ to be $\Delta+1$.

We now prove upper bounds on $\chi_{\mathrm{i} \Sigma}(G)$ for cacti $G$. We start by proving Conjecture 1.2 for even cacti with maximum degree at least 3 .

Theorem 4.5. For every even cactus $G$ with maximum degree $\Delta \geq 3$, we have

$$
\chi_{\mathrm{i} \Sigma}(G) \leq \chi_{\mathrm{i}}(G)=\Delta .
$$

Proof. Recall that for such an even cactus, we have $\chi_{\mathrm{i}}(G)=\Delta$, by Lemma 4.4 . If $G$ has no cycle, then $G$ is a tree in which case the result follows from Theorem 4.2 . Thus, let us assume that $G$ is not a tree. Since $\Delta \geq 3$, we have also that $G$ is not a cycle. We now consider a cycle $C$ of $G$ obtained as follows. While $G$ has vertices with degree 1, we keep on removing them. Since $G$ has cycles, the process finishes with the remaining graph $G^{-}$having minimum degree at least 2 . In $G^{-}$, we consider a cycle $C$ whose all vertices but at most one, say $v^{*}$, have degree 2 (while $v^{*}$ has degree at least 3). Back in $G$, this cycle $C$ is a kind of end-cycle: $v^{*}$ is the vertex of $C$ which is the closest to all other cycles of $G$, if any. At every vertex $v$ of $C$ different from $v^{*}$, there is a tree attached, possibly reduced to $v$, which we denote by $T_{v}$. In what follows below, we consider that every $T_{v}$ is rooted at $v$. In particular, all vertices of $C$ might actually be of degree more than 2 .

For every vertex $r \in V(C) \backslash\left\{v^{*}\right\}$, let us have a look at $T_{r}$. Assume there is an $r$ such that $T_{r}$ is not just $r$, i.e., $T_{r}$ has edges. In that case, let us consider a deepest non-leaf vertex $v$ of $T_{r}$ whose all descendants $u_{1}, \ldots, u_{d}(d \geq 1)$ are its sons (i.e., leaves of $\left.T_{r}\right)$. If $v \neq r$, then the result follows using the arguments used in the proof of Theorem 4.2. Thus, let us assume now that $v=r$, i.e., $T_{r}$ is a star rooted at $r$. In particular, $d(r)=d+2$, where $r$ has two neighbours $v_{1}$ and $v_{2}$ on $C$ (hence of degree at least 2).

Now let $\left(V_{0}, \ldots, V_{\Delta-1}\right)$ be an injective $\Delta$-colouring of $G^{\prime}=G-\left\{u_{1}, \ldots, u_{d}\right\}$, which exists either by Observation 4.3 or Lemma 4.4. Free to relabel the indexes, we may assume that $v_{1} \in V_{1}$ and $v_{2} \in V_{2}$ (since $v_{1}$ and $v_{2}$ must be in different parts, due to $r$ ). Also, we have $r \in V_{x}$, where we might have $x \in\{1,2\}$. By Lemma 3.6 there is an injective $\Delta$-labelling $\ell^{\prime}$ of $G^{\prime}$ where, for every vertex $v \in V_{i}$ different from $r$, we have $c_{\ell^{\prime}}(v) \equiv i \bmod \Delta$. Our goal is to extend $\ell^{\prime}$ to the $r u_{i}$ 's, so that an injective $\Delta$-labelling $\ell$ of $G$ results. To that aim, similarly as in the proof of Theorem 4.2 . we must assign different labels to the $v u_{i}$ 's, in such a way that no $u_{i}$ 's gets the same colour as one of $v_{1}$ and $v_{2}$, and the colour of $r$ becomes $x$ modulo $\Delta$.

Because $d\left(v_{1}\right) \geq 2$ and $v_{1} \in V_{1}$, we have $c_{\ell^{\prime}}\left(v_{1}\right) \geq \Delta+1$. If $d\left(v_{2}\right) \geq 3$, then, because $v_{2} \in V_{2}$, we have $c_{\ell^{\prime}}\left(v_{2}\right) \geq \Delta+2$; in that case, none of the $u_{i}$ 's can have its colour becoming one of $c_{\ell^{\prime}}\left(v_{1}\right)$ and $c_{\ell^{\prime}}\left(v_{2}\right)$, in which case we can freely assign distinct labels to the $r u_{i}$ 's in such a way that they sum up to $\Delta-c_{\ell^{\prime}}(r)$ modulo $\Delta$. Thus, now assume $d\left(v_{2}\right)=2$. If $c_{\ell^{\prime}}\left(v_{2}\right)>\Delta$ then we are done as well, by the same arguments. So assume $c_{\ell^{\prime}}\left(v_{2}\right) \leq \Delta$. Since $c_{\ell^{\prime}}\left(v_{2}\right) \equiv 2 \bmod \Delta$, this means that $c_{\ell^{\prime}}\left(v_{2}\right)=2$, i.e., the two edges incident to $v_{2}$ are labelled 1 . In that situation, we 1 -switch 
$C$, as traversing the closed $(r, r)$-walk that starts from $r$ and goes along $C$ back to $r$. Whatever the length of $C$ is, by Observation 3.3 this does not alter, modulo $\Delta$, the colours of the vertices in $V(C) \backslash\{r\}$. However, this switching changed the labels of the two edges incident to $v_{2}$ to $\Delta$ and 2, respectively, so that now $c_{\ell^{\prime}}\left(v_{2}\right)>\Delta$ and the previous case applies.

Thus, we may now suppose that $V\left(T_{r}\right)=\{r\}$ for every $r \in V(C) \backslash\left\{v^{*}\right\}$, which means that $d(v)=2$ for every vertex $v$ of $C$ different from $v^{*}$. Let us first assume that $C=v_{1} v_{2} v_{3} v_{4} v_{1}$ has length 4 , where $v_{1}=v^{*}$. In that case, let us consider $\left(V_{0}, \ldots, V_{\Delta-1}\right)$ an injective $\Delta$-colouring of $G$, where we assume that $v^{*} \in V_{2}$. By Lemma 3.6, there is an injective $\Delta$-labelling $\ell$ of $G$ where, for every vertex $v \in V_{i}$ different from $v_{3}$, we have $c_{\ell}(v) \equiv i \bmod \Delta$. If $\ell$ is injective, then we are done. Otherwise, it means that $c_{\ell}\left(v^{*}\right)=c_{\ell}\left(v_{3}\right)$, because $v^{*}$ is the only vertex of $G$ that shares a neighbour with $v_{3}$. Since $v^{*} \in V_{2}$ and $d\left(v^{*}\right) \geq 3$, this means that $c_{\ell}\left(v^{*}\right)=c_{\ell}\left(v_{3}\right)=\Delta+2$. In that case, we $\alpha$-switch $C$, as traversing the closed $\left(v_{3}, v_{3}\right)$-walk that starts from $v_{3}$ and goes along $C$ back to $v_{3}$, where $\alpha$ is chosen so that one of the two edges incident to $v_{3}$ has its label becoming 1 (for instance, if $\ell\left(v_{3} v_{2}\right)=x$, then we consider $\alpha=x-1$ and make sure to finish the walk with $\left.v_{3} v_{2}\right)$. Note that this does not modify the colour of the vertices of $C$ modulo $\Delta$, and this makes $c_{\ell}\left(v_{3}\right)$ being at most $\Delta+1$, thus less than $\Delta+2 \leq c_{\ell}\left(v^{*}\right)$.

Now assume $C=v_{0} v_{1} \ldots v_{n-1} v_{0}$ is an even cycle with length $n$ at least 6 , where $v_{0}=v^{*}$. Let us consider $\left(V_{0}, \ldots, V_{\Delta-1}\right)$ an injective $\Delta$-colouring of $G$. We may suppose that $v_{0} \in V_{2}$. By Lemma 3.6. there is an injective $\Delta$-labelling $\ell$ of $G$ where, for every vertex $v \in V_{i}$ different from $v_{2}$, we have $c_{\ell}(v) \equiv i \bmod \Delta$. If $c_{\ell}\left(v_{2}\right) \notin\left\{c_{\ell}\left(v_{0}\right), c_{\ell}\left(v_{4}\right)\right\}$, then we are done, since $v_{0}$ and $v_{4}$ are the only vertices that share a neighbour with $v_{2}$. First assume $c_{\ell}\left(v_{2}\right)=c_{\ell}\left(v_{4}\right)$, and assume $v_{4} \in V_{x}$. In this case, we 1 -switch the path $v_{2} v_{3} v_{4}$ if $v_{4+2} \bmod n \notin V_{x+1}$, while we -1 -switch this path otherwise. Note that this does not modify the colour of $v_{3}$ modulo $\Delta$ in both cases. Regarding $v_{4}$, its colour becomes $x+1$ or $x-1$ modulo $\Delta$, which is different from the colour modulo $\Delta$ of $v_{4+2} \bmod n$. Also, $c_{\ell}\left(v_{4}\right)$ is now different from $c_{\ell}\left(v_{2}\right)$. So now the only possible remaining conflict is $c_{\ell}\left(v_{2}\right)=c_{\ell}\left(v_{0}\right)$, which we can deal with just as in the previous case.

We now prove the counterpart of Theorem 4.5 for odd cacti.

Theorem 4.6. For every odd cactus $G$ with maximum degree $\Delta \geq 3$, we have

$$
\chi_{\mathrm{i} \Sigma}(G) \leq \chi_{\mathrm{i}}(G) \leq \Delta+1 .
$$

Proof. We may assume that $G$ is not a tree or a cycle, as otherwise Theorem 4.2 , 2.5 or 2.6 would apply. Also, we may assume that $\chi_{\mathrm{i}}(G) \equiv 2 \bmod 4$, as otherwise Theorem 3.7 would apply. Since $G$ is not a cycle (and, in particular, verifies $\Delta \geq 3$ ), this means $\chi_{\mathrm{i}}(G) \geq 6$. Set $k=\chi_{\mathrm{i}}(G)$, and let $\left(V_{0}, \ldots, V_{k-1}\right)$ be an injective $k$-colouring. By Lemma 3.5 we can suppose that all $V_{i}$ 's are odd.

First assume that $G$ has a leaf $v$ with unique neighbour $u$. Since $G$ is not a star, $u$ has another neighbour $w$ with $d(w) \geq 2$. We may assume that $w \in V_{1}$. Since $u$ is a common neighbour of $v$ and $w$, we have $v \notin V_{1}$. Now let us consider the vertex-colouring $\left(V_{0}^{\prime}, \ldots, V_{k-1}^{\prime}\right)$ obtained from $\left(V_{0}, \ldots, V_{k-1}\right)$ by moving $v$ to $V_{1}$ (where we assume every $V_{i}^{\prime}$ ' corresponds to the original $V_{i}$ ). Since not all $V_{i}^{\prime}$ 's are odd, by Lemma 3.5 there is a $k$-labelling $\ell$ of $G$ where $c_{\ell}(x) \equiv i \bmod k$ for every $x \in V_{i}^{\prime}$. Note that $\ell$ must be injective, because $\left(V_{0}, \ldots, V_{k-1}\right)$ is an injective colouring, and $c_{\ell}(v)$ must be 1 because $v \in V_{1}^{\prime}$ and $d(v)=1$ while $c_{\ell}(w)$ is more than $k$ since $w \in V_{1}$ and $d(w)>1$.

So we may now assume that $G$ has no leaf. Just as in the proof of Theorem 4.6. let $C$ be an end-cycle. Then all vertices of $C$ but one have degree 2 . If $C$ has length at least 4 , then let us consider a degree-2 vertex $v$ whose two neighbours also have degree 2 . Assume $v \in V_{x}$. Since $\chi_{\mathrm{i}}(G) \geq 6$, we note that, from $\left(V_{0}, \ldots, V_{k-1}\right)$, we can freely move $v$ to another class to get an injective $k$-colouring $\left(V_{0}^{\prime}, \ldots, V_{k-1}^{\prime}\right)$ where not all $V_{i}^{\prime}$ 's are odd. This is because $v$ has at most two vertices at distance 2 , so there are at most two $V_{i}$ 's to which $v$ cannot be moved. Then, from $\left(V_{0}^{\prime}, \ldots, V_{k-1}^{\prime}\right)$, Lemma 3.5 can be applied.

This means that all end-cycles of $G$ are triangles. Let $C=v_{1} v_{2} v_{3} v_{1}$ be an end-cycle of $G$. Note that if we cannot obtain, from $\left(V_{0}, \ldots, V_{k-1}\right)$, an injective $k$-colouring of $G$ by moving $v_{2}$ to another class (in which case we would be done by Lemma 3.5), then this means that $v_{1}$ has degree at least 5. Let then $w$ be a neighbour of $v_{1}$ different from $v_{2}$ and $v_{3}$, such that $w$ does not lie in the same $V_{i}$ as $v_{1}$ (there has to be one such, since any two neighbours of $v_{1}$ cannot lie in the same $\left.V_{i}\right)$. Let us assume that $w \in V_{2}$. Note that, by definition, $w$ is the only neighbour of $v_{1}$ in $V_{2}$, 
and thus the only vertex in $V_{2}$ joined to $v_{2}$ by a path of length 2 . Now let $\left(V_{0}^{\prime}, \ldots, V_{k-1}^{\prime}\right)$ be a $k$-vertex-colouring of $G$ obtained from $\left(V_{0}, \ldots, V_{k-1}\right)$ by moving $v_{2}$ to $V_{2}$ (where we assume every $V_{i}^{\prime}$ ' corresponds to the original $V_{i}$ ). Not all $V_{i}^{\prime}$ 's are odd, so by Lemma 3.5 there is a $k$-labelling $\ell$ of $G$ where $c_{\ell}(v) \equiv i \bmod k$ for every $v \in V_{i}^{\prime}$. If $c_{\ell}\left(v_{2}\right) \neq c_{\ell}(w)$, then $\ell$ is actually injective. So assume this is not the case.

Since $d\left(v_{1}\right) \geq 5$, there must be, in $G$, another end-block $C^{\prime} \neq C$ such that $w$ does not lie on a shortest path from $v_{2}$ to a vertex of $C^{\prime}$. Consider the closed walk $W$ starting from $v_{2}$, going to $v_{1}$ via the edge $v_{2} v_{1}$, then following a shortest path from $v_{1}$ to a vertex $x$ of $C^{\prime}$, then going along $C^{\prime}$ all the way back to $x$, then back to $v_{1}$, and finally traversing the edges $v_{1} v_{3}$ and $v_{3} v_{2}$. Note that $W$ is an even walk not containing $w$, and containing both edges incident to $v_{2}$. By 1-switching or -1 -switching $W$, recall that we do not change the colours of the vertices modulo $k$. One of these two operations, however, has to change the colour of $v_{2}$, which then gets different from $c_{\ell}(w)$. Then $\ell$ becomes injective.

To refine Theorem 4.6 further, a result we miss is a full characterization of the (odd) cacti $G$ with maximum degree $\Delta \geq 3$ and $\chi_{\mathrm{i}}(G)=\Delta+1$. Let us call such a cactus a bad cactus. As mentioned earlier, a cactus is bad as soon as it has a fan vertex. Fan vertices are not enough, however, as there exist bad cacti without fan vertices. For instance, for any even $\Delta \geq 4$, an easy class of bad cacti with maximum degree $\Delta$ is obtained starting from an odd cycle $C$ with length at least 5 , and attaching, to every vertex of $C$, exactly $(\Delta-2) / 2$ pending triangles. This is what we call a fan cycle. Note, in particular, that every fan cycle has no fan vertex.

Again, fan vertices and fan cycles are not sufficient to describe bad cacti. Let us indeed describe a last construction to illustrate this. For the sake of the explanation, we here describe how to construct more bad cacti with maximum degree $\Delta=3$, but the construction can be generalized to any odd $\Delta \geq 3$. Consider a single edge $u v$, and attach a triangle at $v$, so that $v$ has degree 3 . We note that, already for this simple odd cactus $G$, we have $\chi_{\mathrm{i}}(G)=3$, and $u$ and $v$ must receive the same colour in every injective 3-colouring of $G$. We call $G$ a virtual vertex, with root $u$ and subroot $v$. Given a graph $H$ with a vertex $x$, by attaching a virtual vertex $V$ at $x$ we mean identifying $x$ and the root of $V$. Note that if we originally have $\Delta(H) \leq 3$ and $d(x) \leq 2$, then $H$ remains subcubic after the attachment.

There are situations where, upon attaching virtual vertices in a subcubic graph, finding an injective 3-colouring becomes equivalent to finding a distance-2 3-colouring, i.e., a 3-vertex-colouring where no two vertices at distance at most 2 receive the same colour. From this, we can indeed design many subcubic cacti with injective chromatic number 4, and having no fan vertex nor fan cycle. Let us give a simple example. Consider a claw $\left(K_{1,3}\right)$ with center $c$ and three leaves $u_{1}, u_{2}, u_{3}$, and attach a virtual vertex to each $u_{i}$. Then the resulting cactus is subcubic, and in every injective colouring $u_{1}, u_{2}, u_{3}$ must receive distinct colours (since they share $c$ as a common neighbour), while also $c$ must receive a distinct colour. This is because is every virtual vertex the root and subroot get the same colour, while $c$ shares a neighbour with each of the three subroots of the virtual vertices we have attached (namely, the $u_{i}$ 's).

Question 4.7. What is the precise structure of bad cacti?

\subsection{Subcubic graphs}

Before proving our main result in this section, let us start with a structural lemma. In that lemma, we make use of the following terminology. Let $G$ be a graph, and $C=\left(v_{0}, \ldots, v_{n-1}, v_{0}\right)$ be a cycle of $G$. Consider $\vec{C}$ a natural orientation of $C$ being a directed cycle, i.e., either $v_{i} v_{i+1}$ is an arc for every $i \in\{0, \ldots, n-1\}$ or $v_{i} v_{i-1}$ is an arc for every $i \in\{0, \ldots, n-1\}$ (where the operations are understood modulo $n$ ). For every vertex $v_{i}$ of $C$, there is then a successor $v_{i}^{+}$being the one of $v_{i-1}$ or $v_{i+1}$ such that $v_{i} v_{i-1}$ or $v_{i} v_{i+1}$ is the arc in $\vec{C}$. Similarly, for every $v_{i}$ there is a predecessor $v_{i}^{-}$ in $C$. A chord of $C$ is an edge $v_{i} v_{j}$ such that $v_{j}$ is neither a successor nor a predecessor of $v_{i}$. For two vertices $v_{i}, v_{j}$ of $C$, we denote by $\vec{C}\left[v_{i}, v_{j}\right]$ the path $\left(v_{i}, v_{i+1}, \ldots, v_{j}\right)$ of $C$.

Lemma 4.8. Let $G$ be a 2-connected cubic bipartite graph different from $K_{3,3}$. Then there is a vertex $x \in V(G)$ such that $G-x-N(x)$ is connected. 
Proof. First suppose that $G$ has a Hamiltonian cycle $C$ (with any natural orientation $\vec{C}$ ). Recall that $G$ is not $K_{3,3}$. Let $x y$ be a chord of $C$ such that $\vec{C}[x, y]$ is as short as possible. Since $G$ is bipartite, $x^{+} \neq y^{-}$. Let $x^{+} y^{\prime}$ and $x^{\prime} y^{-}$be the chord incident to $x^{+}, y^{-}$, respectively. If $y^{\prime} \neq y^{+}$, then $G-y-N(y)$ is connected, and we are done. So we assume that $y^{\prime}=y^{+}$and similarly, $x^{\prime}=x^{-}$. It follows that $G-y^{+}-N\left(y^{+}\right)$is connected.

Now assume that $G$ is not Hamiltonian. We also assume that $G-v-N(v)$ is disconnected for all $v \in V(G)$. Because it is cubic and 2-connected, by Petersen's Theorem (see [13]) $G$ has a 2 -factor. We choose a 2-factor $F$ of $G$ with number of cycles as small as possible, and we consider that every cycle of $F$ comes with a natural orientation. A link of $G$ is an edge between two distinct cycles of $F$.

Let $x y$ be a link between two cycles $C_{1}, C_{2}$. Recall that $G-x-N(x)$ is disconnected. Let $H_{1}$ be the component of $G-x-N(x)$ containing $C_{1}-\left\{x, x^{+}, x^{-}\right\}$. We choose the link $x y$ such that $H_{1}$ is as large as possible. We conclude that $E\left(\left\{x^{+}, x^{-}\right\},\left\{y^{+}, y^{-}\right\}\right)=\emptyset$, as otherwise we would have a 2-factor with less cycles than $F$.

First assume that $H_{1}$ does not contain $C_{2}-\{y\}$. Let $H_{2}$ be a component of $G-x-N(x)$ containing $C_{2}-\{y\}$. By the 2-connectedness of $G, N_{H_{2}}\left(x^{+}\right) \cup N_{H_{2}}\left(x^{-}\right) \neq \emptyset$. Without loss of generality we assume that $x^{+} x^{\prime} \in E(G)$ with $x^{\prime} \in V\left(H_{2}\right)$. So $x^{\prime} \notin\left\{y^{+}, y^{-}\right\}$. If $x^{\prime} \in V\left(C_{2}\right)$, letting $H_{3}$ be the component of $G-y-N(y)$ containing $C_{2}-\left\{y, y^{+}, y^{+}\right\}$, then $H_{3}$ would contain $H_{1}$, a contradiction. So we assume that $x^{\prime} \notin V\left(C_{2}\right)$. Let $C_{3}$ be the cycle of $F$ containing $x^{\prime}$. It follows that $E\left(\left\{x^{\prime+}, x^{\prime-}\right\},\left\{y^{+}, y^{-}\right\}\right)=\emptyset$, as otherwise we would have a 2-factor with less cycles than $F$. Let $P$ be a path of $H_{2}$ between $x^{\prime}$ and $y$. Then $P$ contains some links. If there is a cycle $C_{4}$ of $F$ such that $\left|E(P) \cap E\left(C_{4}\right)\right| \geq 2$, letting $u v$ be a link contained in $P$ with $u \in V\left(C_{4}\right)$, and $H_{4}$ be the component of $G-u-N(u)$ containing $C_{4}-\left\{u, u^{+}, u^{-}\right\}$, then $H_{4}$ contains $H_{1}$, also a contradiction. It follows that the edges along $P$ are successive links and edges in cycles of $F$. Thus $G$ has a 2 -factor with less cycles than $F$, a contradiction.

Now we assume that $H_{1}$ contains $C_{2}-\{y\}$. Let $H_{2}$ be a component of $G-x-N(x)$ other than $H_{1}$. By the 2-connectedness of $G$, both $N_{H_{2}}\left(x^{-}\right)$and $N_{H_{2}}\left(x^{+}\right)$are not empty. Let $x^{+} x_{1}, x^{-} x_{2} \in$ $E(G)$ with $x_{1}, x_{2} \in V\left(H_{2}\right)$. If $x_{1}$ and $x_{2}$ are contained in a same cycle of $F$, say $C_{3}$, letting $H_{3}$ be the component of $G-x_{1}-N\left(x_{1}\right)$ containing $C_{3}-\left\{x_{1}, x_{1}^{+}, x_{1}^{+}\right\}$, then $H_{3}$ would contain $H_{1}$, a contradiction. So we assume that $x_{1} \in V\left(C_{3}\right)$ and $x_{2} \in V\left(C_{4}\right)$, where $C_{3}$ and $C_{4}$ are distinct cycles of $F$. Let $P$ be a path of $H_{2}$ between $x_{1}$ and $x_{2}$. Then $P$ contains some links. If there is a cycle $C_{5}$ of $F$ such that $\left|E(P) \cap E\left(C_{5}\right)\right| \geq 2$, letting $u v$ be a link contained in $P$ with $u \in V\left(C_{5}\right)$, and $H_{5}$ be the component of $G-u-N(u)$ containing $C_{5}-\left\{u, u^{+}, u^{-}\right\}$, then $H_{5}$ contains $H_{1}$, a contradiction. It follows that the edges along $P$ are successive links and edges in cycles of $F$. Thus $x x^{+} x_{1} P x_{2} x^{-} x$ is an odd cycle, a contradiction.

We will also need a counterpart of the results in Section 3 when the graph is bipartite and we are considering a 3-vertex-colouring of it. For a bipartite graph $G$ with bipartition $(X, Y)$, we say that a 3-vertex-colouring $\left(V_{0}, V_{1}, V_{2}\right)$ is good if $\left|X_{1}\right|-\left|X_{2}\right| \equiv\left|Y_{1}\right|-\left|Y_{2}\right| \bmod 3$, where $X_{i}=V_{i} \cap X$ and $Y_{i}=V_{i} \cap Y$ for every $i \in\{0,1,2\}$.

Lemma 4.9. Let $G$ be a bipartite graph with bipartition $(X, Y)$, and $\left(V_{0}, V_{1}, V_{2}\right)$ be a 3-vertexcolouring (with no specific properties) of $G$, where $V_{i}=X_{i} \cup Y_{i}$, for $X_{i} \subseteq X$ and $Y_{i} \subseteq Y$, for every $i \in\{0,1,2\}$. Then $G$ admits a 3-labelling $\ell$ such that, for every $i \in\{0,1,2\}$ and every vertex $v \in V_{i}$, we have $c_{\ell}(v) \equiv i \bmod 3$ if and only if $\left(V_{0}, V_{1}, V_{2}\right)$ is good.

Proof. Again, we can equivalently look for a $\{0,1,2\}$-labelling $\ell$ with the desired properties. Suppose we have a labelling $\ell$ satisfying the assertion. Then

$$
\sum_{x \in X} c_{\ell}(x)=\sum_{e \in E(G)} \ell(e)=\sum_{y \in Y} c_{\ell}(y)
$$

Note that $\sum_{x \in X} c_{\ell}(x) \equiv\left|X_{1}\right|-\left|X_{2}\right| \bmod 3$ and $\sum_{y \in Y} c_{\ell}(y) \equiv\left|Y_{1}\right|-\left|Y_{2}\right| \bmod 3$. So we have that $\left|X_{1}\right|-\left|X_{2}\right| \equiv\left|Y_{1}\right|-\left|Y_{2}\right| \bmod 3$.

We now prove the converse direction. Suppose that $\left|X_{1}\right|-\left|X_{2}\right| \equiv\left|Y_{1}\right|-\left|Y_{2}\right| \bmod 3$. We design a labelling $\ell$ satisfying the assertion. First label every edge 0 , so that every vertex, for now, has colour 0 modulo 3 . Now, take three vertices from one of the sets $X_{1}, X_{2}, Y_{1}, Y_{2}$, say 
$x, x^{\prime}, x^{\prime \prime} \in X_{i}$, and one arbitrary vertex $v \in V(G) \backslash\left\{x, x^{\prime}, x^{\prime \prime}\right\}$. Let $P, P^{\prime}, P^{\prime \prime}$ be three paths from $x, x^{\prime}, x^{\prime \prime}$, respectively, to $v$. Note that the lengths of these three paths have the same parity. Let us now $i$-switch the paths $P, P^{\prime}, P^{\prime \prime}$. As seen earlier, the colour of $x, x^{\prime}, x^{\prime \prime}$ becomes $i$ modulo 3 , while the colour of all other vertices remains the same modulo 3. Repeating this operation, we can get to a situation where at most two vertices in each of $X_{1}, X_{2}, Y_{1}, Y_{2}$ does not have the desired colour modulo 3 .

If there remain defective vertices in both $X_{1}$ and $X_{2}$, or in both $Y_{1}$ and $Y_{2}$, say $x \in X_{1}$ and $x^{\prime} \in X_{2}$, then we choose a path $P$ from $x$ to $x^{\prime}$. So $P$ has even length. Let us 1 -switch $P$. The colour of $x$ and $x^{\prime}$ becomes 1 and 2 modulo 3, respectively, while all other vertices keep the same colour modulo 3. Repeating this operation, we get to the a situation where either $X_{1}$ or $X_{2}$ is empty, and either $Y_{1}$ or $Y_{2}$ is empty.

If there remain defective vertices in $X_{i}$ (for $i \in\{1,2\}$ ), then there are the same number of defective vertices in $Y_{i}$ (recall that $\left|X_{1}\right|-\left|X_{2}\right| \equiv\left|Y_{1}\right|-\left|Y_{2}\right| \bmod 3$ ). For two such remaining vertices $x \in X_{i}$ and $y \in Y_{i}$, we choose a path $P$ from $x$ to $y$. So $P$ has odd length. We here $i$-switch $P$. Again, the colour of $x$ and $y$ becomes $i$ modulo 3, while all other vertices keep the same colour modulo 3. The resulting labelling is now as desired.

We are now ready to prove our main result in this section.

Theorem 4.10. For every graph $G$ with $\Delta(G)=3$ and $\chi_{\mathrm{i}}(G)=3$, we have $\chi_{\mathrm{i} \Sigma}(G) \leq 3=\Delta(G)$.

Proof. Let $G$ be a connected graph with $\chi_{\mathrm{i}}(G)=3$. So $G$ is subcubic. If $G$ is not bipartite, then the result follows from Lemma 3.4. So let us assume $G$ is bipartite, and let $(X, Y)$ denote the bipartition of $G$. Towards a contradiction, assume $G$ is a counterexample to the claim. If $G$ has a good injective 3-vertex-colouring, then we can deduce an injective 3-labelling of $G$ by Lemma 4.9 . a contradiction. So we assume that every injective 3 -vertex-colouring of $G$ is not good.

Claim 4.11. Let $\left(V_{0}, V_{1}, V_{2}\right)$ be an injective 3-vertex-colouring of $G$, where $X_{i}=V_{i} \cap X$ and $Y_{i}=V_{i} \cap Y$ for every $i \in\{0,1,2\}$. Then, up to symmetry, all $X_{i}$ 's have the same size modulo 3 , and all $Y_{i}$ 's have pairwise distinct size modulo 3.

Proof of the claim. If $\left|X_{j}\right| \equiv\left|X_{k}\right| \bmod 3$ for some $0 \leq j<k \leq 3$ and $\left|Y_{s}\right| \equiv\left|Y_{t}\right| \bmod 3$ for some $0 \leq s<t \leq 3$, then $\left(X_{3-j-k} \cup Y_{3-s-t}, X_{j} \cup Y_{s}, X_{k} \cup Y_{t}\right)$ is a good injective 3-vertex-colouring of $G$, and Lemma 4.9 raises contradiction. Thus without loss of generality we assume that $Y_{0}, Y_{1}, Y_{2}$ have pairwise distinct size modulo 3 . Now if $\left|X_{j}\right| \not \equiv\left|X_{k}\right| \bmod 3$ for some $0 \leq j<k \leq 3$, then either $\left|X_{j}\right|-\left|X_{k}\right| \equiv\left|Y_{1}\right|-\left|Y_{2}\right| \bmod 3$ or $\left|X_{k}\right|-\left|X_{j}\right| \equiv\left|Y_{1}\right|-\left|Y_{2}\right| \bmod 3$. Thus $G$ has again a good injective 3 -vertex-colouring, and Lemma 4.9 gets us to a contradiction. Thus we must have $\left|X_{0}\right| \equiv\left|X_{1}\right| \equiv\left|X_{2}\right| \bmod 3$.

Claim 4.12. Let $\left(V_{0}, V_{1}, V_{2}\right)$ be a good (non-injective) 3-vertex-colouring of $G$, where $X_{i}=V_{i} \cap X$ and $Y_{i}=V_{i} \cap Y$ for every $i \in\{0,1,2\}$. Then, for any two permutations $(i, j, k)$ and $(r, s, t)$ of $(0,1,2)$, the 3-vertex-colouring $\left(X_{i} \cup Y_{r}, X_{j} \cup Y_{s}, X_{k} \cup Y_{t}\right)$ is also good.

Proof of the claim. By Claim 4.11, we have $|X| \equiv|Y| \equiv 0 \bmod 3$. It can be checked that $\left|X_{0}\right|-\left|X_{1}\right| \equiv\left|X_{1}\right|-\left|X_{2}\right| \equiv\left|X_{2}\right|-\left|X_{0}\right| \bmod 3$ and $\left|Y_{0}\right|-\left|Y_{1}\right| \equiv\left|Y_{1}\right|-\left|Y_{2}\right| \equiv\left|Y_{2}\right|-\left|Y_{0}\right| \bmod 3$. Thus the assertion follows from the definition of good 3-vertex-colourings.

Claim 4.13. $\delta(G) \geq 2$.

Proof of the claim. Suppose $G$ has a vertex, say $x \in X$, of degree 1 . Let $y$ be the unique neighbour of $x$, and $x^{\prime}$ be a neighbour of $y$ with $d\left(x^{\prime}\right) \geq 2$ (recall that $|X| \equiv|Y| \equiv 0 \bmod 3$, implying that $G$ is not a star). Let $\left(V_{0}, V_{1}, V_{2}\right)=\left(X_{0} \cup Y_{0}, X_{1} \cup Y_{1}, X_{2} \cup Y_{2}\right)$ be an injective 3-colouring of $G$ with $x \in X_{0}$ and $x^{\prime} \in X_{1}$. By moving $x$ from $X_{0}$ to $X_{1}$, we get a new 3-colouring $\left(V_{0}^{\prime}, V_{1}^{\prime}, V_{2}^{\prime}\right)=\left(\left(X_{0} \backslash\{x\}\right) \cup Y_{0},\left(X_{1} \cup\{x\}\right) \cup Y_{1}, X_{2} \cup Y_{2}\right)$ of $G$. By relabelling, if necessary, some of the parts, note that this colouring is good. By Lemma 4.9, there exists a 3-labelling $\ell$ of $G$ such that $c_{\ell}(v) \equiv i \bmod 3$ for every vertex $v \in V_{i}^{\prime}$. Clearly $c_{\ell}(x)=1$ and $c_{\ell}\left(x^{\prime}\right) \geq 4$. It follows that $c_{\ell}$ is an injective colouring of $G$, thus that $\ell$ is injective, a contradiction. 
Claim 4.14. Let $u \in V(G)$ be a vertex of $G$ such that each edge incident to $u$ is contained in a cycle, and let $\left(V_{0}, V_{1}, V_{2}\right)$ be a good (non-injective) 3-colouring of $G$, where $u \in V_{i}$ for some $i \in\{0,1,2\}$. Set $S=\left\{u_{1}, \ldots, u_{k}\right\}=\left\{v \in V_{i}: N(u) \cap N(v) \neq \emptyset\right.$ and $\left.d(v)=d(u)\right\}$. Suppose that

1. every two vertices $v_{1}, v_{2} \in V(G) \backslash\{u\}$ with $N\left(v_{1}\right) \cap N\left(v_{2}\right) \neq \emptyset$ are not contained in a same colour class; and

2. there exist cycles $C_{1}, \ldots, C_{k}$ avoiding $u$, and there exist edges $e_{1}, \ldots, e_{k}$ incident to $u_{1}, \ldots, u_{k}$, respectively, such that $e_{i} \in E\left(C_{j}\right)$ if and only if $i=j$.

Then $G$ has an injective 3-labelling $\ell$.

Proof of the claim. Here and throughout, we call a 3-colouring of $G$ with the properties above a better colouring (associated with $u$ ). First suppose that $d(u)=2$. By Claim 4.12, we can assume that $u \in V_{2}$. By Lemma 4.9, there exist a 3-labelling $\ell$ of $G$ such that $c_{\ell}(v) \equiv i \bmod 3$ for every vertex $v \in V_{i}$. Thus every two vertices of $V(G) \backslash\{u\}$ having a common neighbour get distinct colours modulo 3 .

Let $e$ be an edge incident with $u$ and $C$ be a cycle containing $e$. Recall that $G$ is bipartite and thus $C$ is an even cycle. Let us 1 -switch $C$, as initiating the closed walk from any vertex. This does not change the colours modulo 3 of the vertices. We repeat this operation until $\ell(e)=1$ holds. Since $d(u)=2$ and $c_{\ell}(u) \equiv 2 \bmod 3$, we deduce that $c_{\ell}(u)=2$.

For every vertex $u_{i} \in S$, we repeatedly 1-switch $C_{i}$ until $\ell\left(e_{i}\right) \geq 2$ holds. It follows that $c_{\ell}\left(u_{i}\right) \geq 5$ for all $u_{i} \in S$. For every vertex $v \in V_{2}$ with $N(u) \cap N(v) \neq \emptyset$ and $d(v)=3$, it is clear that $c_{\ell}(v) \geq 5$. Thus $c_{\ell}$ is an injective colouring of $G$, and $\ell$ an injective 3-labelling, a contradiction

Now we suppose that $d(u)=3$, and we start again from a 3-labelling $\ell$ of $G$ such that $c_{\ell}(v) \equiv$ $i$ mod 3 for every vertex $v \in V_{i}$ (a such one exists by Lemma 4.9). Let $e_{1}, e_{2}, e_{3}$ be edges incident to $u$, and $C$ be a cycle containing $e_{1}$. Without loss of generality we assume that $C$ contains $e_{2}$. Let $C^{\prime}$ be a cycle containing $e_{3}$. Without loss of generality we assume that $C^{\prime}$ contains $e_{2}$. So $e_{1}, e_{2} \in E(C)$ and $e_{2}, e_{3} \in E\left(C^{\prime}\right)$. We repeatedly 1 -switch $C$ and $C^{\prime}$ until $\ell\left(e_{1}\right)=\ell\left(e_{3}\right)=3$. Because $c_{\ell}(u) \equiv 0 \bmod 3$, we deduce that $c_{\ell}(u)=9$.

For every vertex $u_{i} \in S$, we repeatedly 1-switch $C_{i}$ until $\ell\left(e_{i}\right) \leq 2$. It follows that $c_{\ell}\left(u_{i}\right) \leq 6$ for every $u_{i} \in S$. For every vertex $v \in V_{0}$ with $N(u) \cap N(v) \neq \emptyset$ and $d(v)=2$, it is clear that $c_{\ell}(v) \leq 6$. Thus $c_{\ell}$ is an injective colouring of $G$, and $\ell$ an injective 3-labelling, a contradiction. $\diamond$

We now distinguish two main cases, depending on whether $G$ is 2-connected or separable (i.e., has articulation vertices).

Case 1: $G$ is separable.

Let $B$ be an end-block of $G$, and $u$ be the cut-vertex of $G$ contained in $B$. Recall that $\delta(G) \geq 2$, implying that $B$ is 2 -connected. In particular, we have $d(u)=3$. Let $u^{\prime}$ be the unique neighbour of $u$ outside $B$. Then $u u^{\prime}$ is a cut-edge of $G$. We claim that there is some vertex $v \in V(B)$ with $d(v)=2$. This is because otherwise we would have $\sum_{x \in V(B) \cap X} d_{B}(x) \not \equiv \sum_{y \in V(B) \cap Y} d_{B}(y) \bmod 3$, a contradiction. In particular, all vertices in $B$ have degree 3 except $u$ which has degree 2 ; then the size of $B$ can be expressed both as the sum of the degrees of the vertices in $V(B) \cap X$ or as the sum of the degrees of the vertices in $V(B) \cap Y$, while these two quantities are not equal.

First assume that $B$ is a cycle $B=\left(u, v_{1}, u_{1}, v_{2}, u_{2}, \ldots, u\right)$ (we might have $u=u_{2}$ ). Let $\left(V_{0}, V_{1}, V_{2}\right)$ be an injective 3-colouring of $G$ with, say, $u_{1} \in V_{0}$ and $u_{2} \in V_{1}$. By moving $u_{1}$ from $V_{0}$ to $V_{2}$, we get a 3 -colouring $\left(V_{0}^{\prime}, V_{1}^{\prime}, V_{2}^{\prime}\right)=\left(V_{0} \backslash\left\{u_{1}\right\}, V_{1}, V_{2} \cup\left\{u_{1}\right\}\right)$. Note that the only vertices having a common neighbour with $u_{1}$ are $u$ and $u_{2}$. Since $u$ and $u_{1}$ have distinct degree, and $u_{1}$ and $u_{2}$ do not belong to a same $V_{i}^{\prime}$, we get that $\left(V_{0}^{\prime}, V_{1}^{\prime}, V_{2}^{\prime}\right)$ is a better 3 -colouring associated with $u_{1}$. Now Claim 4.14 applies, a contradiction. So we assume that $B$ is not a cycle, i.e., there are some vertices in $V(B) \backslash\{u\}$ with degree 3 .

Now we assume that $B$ has two adjacent vertices both of degree 2. By consider a longest path with all internal vertices of degree 2 in $B$, we can find a path, say $\left(y, x, y^{\prime}\right)$, such that $d(y)=3$ and $d(x)=d\left(y^{\prime}\right)=2$. Set $N(y)=\left\{x, x_{1}, x_{2}\right\}$ and $N\left(y^{\prime}\right)=\left\{x, x_{1}^{\prime}\right\}$. Let $\left(V_{0}, V_{1}, V_{2}\right)$ be an injective 3-colouring with $x \in V_{0}$ and $x_{1}^{\prime} \in V_{1}$. By moving $x$ from $X_{0}$ to $X_{2}$, we get a new 3-colouring $\left(V_{0}^{\prime}, V_{1}^{\prime}, V_{2}^{\prime}\right)=\left(V_{0} \backslash\{x\}, V_{1}, V_{2} \cup\{x\}\right)$. Note that the only vertices in $V_{2}^{\prime}$ that have a common neighbour with $x$ is one vertex of $x_{1}, x_{2}$. Since $\left(x_{1}, y, x_{2}\right)$ (or $\left(x_{1}, y, x\right)$ if $u=y$ ) is contained in 
a cycle avoiding $x$, we get that $\left(V_{0}^{\prime}, V_{1}^{\prime}, V_{2}^{\prime}\right)$ is a better 3 -colouring of $G$ associated with $u_{1}$, and Claim 4.14 raises a contradiction. It follows that the set of vertices in $B$ of degree 2 must be an independent set.

Suppose now that there are two adjacent vertices both of degree 3. Let $H$ be the component of the subgraph induced by $\left\{v \in V(B): d_{G}(v)=3\right\}$, with $|V(H)| \geq 2$. Let $u_{1} v_{1} \in E(H, B-H)$. So $d\left(v_{1}\right)=2$ and set $N\left(v_{1}\right)=\left\{u_{1}, u_{2}\right\}$. We claim that we can choose $u_{1} v_{1}$ such that $u \neq u_{2}$. Suppose that for every edge $u_{1} v_{1} \in E(H, B-H)$, the neighbour of $v_{1}$ other than $u_{1}$ is $u$. It follows that $v_{1}$ has one neighbour outside $H$ (which is $u$ ) and that there are exactly two edges between $H$ and $B-H$. But now $\sum_{x \in V(H) \cap X} d_{H}(x) \not \equiv \sum_{y \in V(H) \cap Y} d_{H}(y) \bmod 3$, a contradiction due to the number of edges $B$.

Now let $\left(y, x, y^{\prime}, x^{\prime}\right)$ be a path in $B$ such that $d(x)=2, d(y)=d\left(y^{\prime}\right)=d\left(x^{\prime}\right)=3$ and $u \neq y$. Set $N(y)=\left\{x, x_{1}, x_{2}\right\}$ and $N\left(y^{\prime}\right)=\left\{x, x^{\prime}, x_{1}^{\prime}\right\}$ (we might have $x^{\prime} \in N(y)$ ). Let $\left(V_{0}, V_{1}, V_{2}\right)$ be an injective 3-colouring with $x \in V_{0}, x_{1}^{\prime} \in V_{1}$ and $x^{\prime} \in V_{2}$. By moving $x$ from $X_{0}$ to $X_{2}$, we get a new 3-colouring $\left(V_{0}^{\prime}, V_{1}^{\prime}, V_{2}^{\prime}\right)=\left(V_{0} \backslash\{x\}, V_{1}, V_{2} \cup\{x\}\right)$. Note that the only vertices in $V_{2}^{\prime}$ that have a common neighbour with $x$ and possibly having the same degree with $x$ are one of $x_{1}, x_{2}$. Since $\left(x_{1}, y, x_{2}\right)$ is contained in a cycle avoiding $x$, we get that $\left(V_{0}^{\prime}, V_{1}^{\prime}, V_{2}^{\prime}\right)$ is a better 3-colouring of $G$ associated with $x$. Claim 4.14 gives another contradiction. It follows that the set of vertices in $B$ of degree 3 must be an independent set.

Now let $x \in V(B) \cap X$ be a vertex with $d(x)=3$. Set $N(x)=\left\{y_{1}, y_{2}, y_{3}\right\}$ and $N\left(y_{i}\right)=\left\{x, x_{i}\right\}$ for $i \in\{1,2,3\}$. Let $\left(V_{0}, V_{1}, V_{2}\right)=\left(X_{0} \cup Y_{0}, X_{1} \cup Y_{1}, X_{2} \cup Y_{2}\right)$ be an injective 3-colouring with $x \in X_{1}$. So $x_{1}, x_{2}, x_{3} \in X_{0} \cup X_{2}$. If necessary, we relabel $X_{0}$ and $X_{1}$ so that $\left|X_{0} \cap\left\{x_{1}, x_{2}, x_{3}\right\}\right| \leq 1$. If $\left|X_{0} \cap\left\{x_{1}, x_{2}, x_{3}\right\}\right|=0$, then by moving $x$ from $X_{1}$ to $X_{0}$, we get a good 3-colouring of $G$, thus a contradiction by Claim 4.12. So we assume without loss of generality that $x_{1} \in X_{0}$ and $x_{2}, x_{3} \in X_{2}$. By moving $x$ from $X_{1}$ to $X_{0}$, we get a 3-colouring $\left(V_{0}^{\prime}, V_{1}^{\prime}, V_{2}^{\prime}\right)=\left(\left(X_{0} \cup\{x\}\right) \cup Y_{0}, X_{1} \backslash\{x\} \cup Y_{1}, X_{2} \cup\right.$ $\left.Y_{2}\right)$ of $G$. If necessary, we can relabel $X_{1} \backslash\{x\}, X_{2}$ so that $\left(V_{0}^{\prime}, V_{1}^{\prime}, V_{2}^{\prime}\right)$ is a good 3-colouring. If necessary, we relabel some of $Y_{0}^{\prime}, Y_{1}^{\prime}, Y_{2}^{\prime}$ so that $y_{1} \in Y_{1}$. By Lemma 4.9 there exists a 3-labelling $\ell$ of $G$ such that $c_{\ell}(v) \equiv i \bmod 3$ for every vertex $v \in V_{i}^{\prime}$. Thus every two vertices in $V(G) \backslash\{x\}$ having a common neighbour get distinct colours modulo 3 .

Now let $C$ be a cycle containing $\left(y_{1}, x, y_{2}\right)$, and $C^{\prime}$ be a cycle containing $\left(y_{2}, x, y_{3}\right)$ (which exists since $B$ is 2 -connected, so every edge is contained in two cycles). We repeatedly 1 -switch $C$ and $C^{\prime}$ so that $\ell\left(x y_{1}\right)=\ell\left(x y_{2}\right)=\ell\left(x y_{3}\right)=1$ (first make $\ell\left(x y_{1}\right)=1$, and the make $\ell\left(x y_{3}\right)=1$; now, since $x \in X_{0}$ we have $\left.\ell\left(x y_{2}\right)=1\right)$. Thus we have $c_{\ell}(x)=3$. Recall that $y_{1} \in Y_{1}$ and $d\left(y_{1}\right)=2$. We have $\ell\left(y_{1} x_{1}\right)=3$, implying that $c_{\ell}\left(x_{1}\right) \geq 6$, and $c_{\ell}$ is a injective colouring of $G$, and $\ell$ an injective labelling, a contradiction.

Case 2.1: $G$ is 2-connected and $\delta(G)=2$.

Recall that $\Delta(G)=3$. First assume that $G$ has two adjacent vertices both of degree 2. By considering a longest path with all internal vertices of degree 2 , we can find a path $\left(y, x, y^{\prime}\right)$ such that $d(y)=3$ and $d(x)=d\left(y^{\prime}\right)=2$. Set $N(y)=\left\{x, x_{1}, x_{2}\right\}$ and $N\left(y^{\prime}\right)=\left\{x, x_{1}^{\prime}\right\}$. Let $\left(V_{0}, V_{1}, V_{2}\right)$ be an injective 3-colouring of $G$ with $x \in V_{0}$ and $x_{1}^{\prime} \in V_{1}$. By moving $x$ from $X_{0}$ to $X_{2}$, we get a better 3-colouring $\left(V_{0}^{\prime}, V_{1}^{\prime}, V_{2}^{\prime}\right)=\left(V_{0} \backslash\{x\}, V_{1}, V_{2} \cup\{x\}\right)$ associated with $x$ (the only vertex in $V_{2}^{\prime}$ with the same degree as $x$ (if any) is exactly one in $\left\{x_{1}, x_{2}\right\}$, while $x_{1}, y, x_{2}$ are contained in a cycle avoiding $x$ ), and we get a contradiction through the use of Claim 4.14. It follows that the set of vertices of degree 2 must be an independent set.

Suppose now that there are two adjacent vertices both of degree 3 . So there is a path $\left(y, x, y^{\prime}, x^{\prime}\right)$ such that $d(x)=2$ and $d(y)=d\left(y^{\prime}\right)=d\left(x^{\prime}\right)=3$. Set $N(y)=\left\{x, x_{1}, x_{2}\right\}$ and $N\left(y^{\prime}\right)=\left\{x, x^{\prime}, x_{1}^{\prime}\right\}$. Let $\left(V_{0}, V_{1}, V_{2}\right)$ be an injective 3-colouring of $G$ with $x \in V_{0}, x_{1}^{\prime} \in V_{1}$ and $x^{\prime} \in V_{2}$. By moving $x$ from $X_{0}$ to $X_{2}$, we get a better 3-colouring $\left(V_{0}^{\prime}, V_{1}^{\prime}, V_{2}^{\prime}\right)=\left(V_{0} \backslash\{x\}, V_{1}, V_{2} \cup\{x\}\right)$ associated with $x$ (by the same reasons as earlier). So that Claim 4.14 does not yield a contradiction, the set of vertices of degree 3 must be an independent set.

Now let $x \in X$ be a vertex with $d(x)=3$. Set $N(x)=\left\{y_{1}, y_{2}, y_{3}\right\}$ and $N\left(y_{i}\right)=\left\{x, x_{i}\right\}$ for $i \in\{1,2,3\}$. Let $\left(V_{0}, V_{1}, V_{2}\right)=\left(X_{0} \cup Y_{0}, X_{1} \cup Y_{1}, X_{2} \cup Y_{2}\right)$ be an injective 3 -colouring with $x \in X_{1}$. So $x_{1}, x_{2}, x_{3} \in X_{0} \cup X_{2}$. If necessary, we relabel $X_{0}, X_{1}$ so that $\left|X_{0} \cap\left\{x_{1}, x_{2}, x_{3}\right\}\right| \leq 1$. If $\mid X_{0} \cap$ $\left\{x_{1}, x_{2}, x_{3}\right\} \mid=0$, then by moving $x$ from $X_{1}$ to $X_{0}$, we get a good 3-colouring of $G$, a contradiction by Claim 4.12. So we assume without loss of generality that $x_{1} \in X_{0}$ and $x_{2}, x_{3} \in X_{2}$. By moving $x$ from $X_{1}$ to $X_{0}$, we get a new 3 -colouring $\left(V_{0}^{\prime}, V_{1}^{\prime}, V_{2}^{\prime}\right)=\left(\left(X_{0} \cup\{x\}\right) \cup Y_{0}, X_{1} \backslash\{x\} \cup Y_{1}, X_{2} \cup Y_{2}\right)$. 


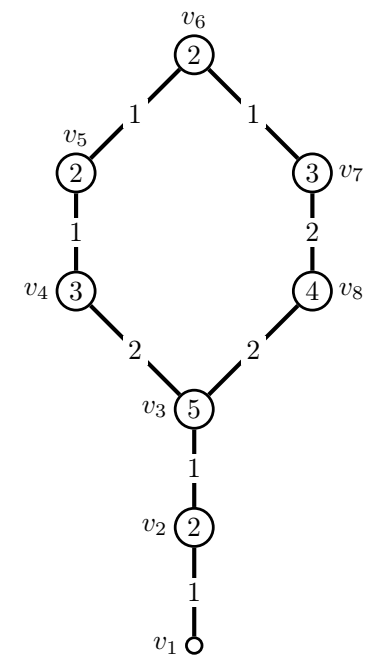

Figure 1: The gadget $T$, and an injective 2-labelling of $T$. An integer in a vertex indicates its colour by the depicted labelling.

If necessary, we can relabel $X_{1} \backslash\{x\}, X_{2}$. Then this results in a good 3-colouring. If necessary, we relabel some of $Y_{0}^{\prime}, Y_{1}^{\prime}, Y_{2}^{\prime}$ so that $y_{1} \in Y_{1}$. By Lemma 4.9, there is a 3-labelling $\ell$ of $G$ such that $c_{\ell}(v) \equiv i \bmod 3$ for every vertex $v \in V_{i}^{\prime}$. Thus every two vertices in $V(G) \backslash\{x\}$ having a common neighbour get distinct colours modulo 3 .

Let $C$ be a cycle of $G$ containing $\left(y_{1}, x, y_{2}\right)$, and $C^{\prime}$ be a cycle containing $\left(y_{2}, x, y_{3}\right)$. Just as in the last case of Case 1, we repeatedly 1-switch $C$ and $C^{\prime}$ until $\ell\left(x y_{1}\right)=\ell\left(x y_{2}\right)=\ell\left(x y_{3}\right)=1$. Thus we have $c_{\ell}(x)=3$. Recall that $y_{1} \in Y_{1}$ and $d\left(y_{1}\right)=2$. We have that $\ell\left(y_{1} x_{1}\right)=3$, implying that $c_{\ell}\left(x_{1}\right) \geq 6$ and $c_{\ell}$ is a proper injective colouring of $G$, and thus $\ell$ an injective 3-labelling, a contradiction.

Case 2.2: $G$ is 2-connected and cubic

Note that $G \neq K_{3,3}$ since $G$ has no good 3-colouring. By Lemma 4.8, there is a vertex $x \in X$ such that $G-x-N(x)$ is connected. Set $N(x)=\left\{y_{1}, y_{2}, y_{3}\right\}$ and $N\left(y_{i}\right)=\left\{x, x_{i}, x_{i}^{\prime}\right\}$ where $i \in\{1,2,3\}$ (possibly $x_{i}=x_{j}$ or $x_{i}=x_{j}^{\prime}$ or $x_{i}^{\prime}=x_{j}^{\prime}$ for some $1 \leq i<j \leq 3$ ). Let $\left(V_{0}, V_{1}, V_{2}\right)$ be an injective 3-colouring with $x \in V_{0}$. Thus $x_{i}, x_{i}^{\prime} \in X_{0} \cup X_{2}$ for $i \in\{1,2,3\}$. Without loss of generality we assume that $x_{i} \in V_{1}$ and $x_{i}^{\prime} \in V_{2}$. By moving $x$ from $X_{0}$ to $X_{1}$, we get a new 3 -colouring $\left(V_{0}^{\prime}, V_{1}^{\prime}, V_{2}^{\prime}\right)=\left(V_{0} \backslash\{x\}, V_{1} \cup\{x\}, V_{2}\right)$.

Let $P_{i}$ be a path of $G-x-N(x)$ between $x_{i}$ and $x_{i}^{\prime}$ (recall that $G-x-N(x)$ is connected), and let $C_{i}=\left(y_{i}, x_{i}, P_{i}, x_{i}^{\prime}, y_{i}\right)$. Thus the edge $x_{i} y_{i}$ is not contained in the cycle $C_{j}$ for $i \neq j$. It follows that $\left(V_{0}^{\prime}, V_{1}^{\prime}, V_{2}^{\prime}\right)$ is a better 3 -colouring associated of $G$ with $x$. Now Claim 4.14 gives us our final contradiction.

\section{On the complexity of determining $\chi_{\mathrm{i} \Sigma}$}

In this section, we investigate the hardness of determining $\chi_{\mathrm{i} \Sigma}(G)$ for a given graph $G$. Our main result below, Theorem 5.3 is that deciding whether $\chi_{\mathrm{i} \Sigma}(G) \leq 2$ holds is an NP-complete problem, even when restricted to instances where $G$ is bipartite. Note that this is contrasting with the problem of deciding whether $\chi_{\Sigma}(G) \leq 2$ holds for a given bipartite graph $G$, which was shown to be in $P$ (see [16]).

Before proceeding with the proof, we first need to introduce some gadgets. The 1-gadget $T$ is depicted in Figure 1. In what follows, we deal with its vertices and edges through the notation from the figure. The vertex $v_{1}$ of $T$ is its root, the vertex $v_{2}$ is its subroot, while the unique edge incident to $v_{1}$ is its root edge. The important property is that all injective 2-labellings of $T$ assign the same label to the root edge.

Lemma 5.1. Let $\ell$ be an injective 2-labelling of $T$. Then: 
- $\ell\left(v_{1} v_{2}\right)=1$;

- $c_{\ell}\left(v_{3}\right) \leq 6$;

- $c_{\ell}\left(v_{2}\right) \leq 3$.

Furthermore, injective 2-labellings of $T$ do exist.

Proof. The last two items follow from the degree of $v_{2}$ and $v_{3}$, because of the first item, and because we are considering 2-labellings. To see that the last part of the statement is true as well, we provide an example of injective 2-labelling of $T$ in Figure 1. So we just need to focus on proving the first item.

Let us investigate how $\ell$ behaves in $T$. We want to show that we must have $\ell\left(v_{1} v_{2}\right)=1$, so let us assume to the contrary that $\ell\left(v_{1} v_{2}\right)=2$. We distinguish the two possible values as $\ell\left(v_{2} v_{3}\right)$ separately.

- Assume $\ell\left(v_{2} v_{3}\right)=1$. Then $c_{\ell}\left(v_{2}\right)=3$. First, we note that if $\ell\left(v_{3} v_{4}\right)=\ell\left(v_{3} v_{8}\right)$, then, so that $c_{\ell}\left(v_{4}\right)$ and $c_{\ell}\left(v_{8}\right)$ are different from $c_{\ell}\left(v_{2}\right)$ (which is required, since $v_{3}$ is a common neighbour of $v_{2}$ and $\left.v_{4}, v_{8}\right)$, we must actually have $\ell\left(v_{3} v_{4}\right)=\ell\left(v_{3} v_{8}\right)=\ell\left(v_{4} v_{5}\right)=\ell\left(v_{8} v_{7}\right)$. Then $c_{\ell}\left(v_{4}\right)=c_{\ell}\left(v_{8}\right)$ while $v_{3}$ is a common neighbour of $v_{4}$ and $v_{8}$, a contradiction. So we must have, say $\ell\left(v_{3} v_{4}\right)=1$ and $\ell\left(v_{3} v_{8}\right)=2$, and thus $c_{\ell}\left(v_{3}\right)=4$. By the same argument as above, we must have $\ell\left(v_{4} v_{5}\right)=1$ and $\ell\left(v_{8} v_{7}\right)=2$, and thus $c_{\ell}\left(v_{4}\right)=2$ and $c_{\ell}\left(v_{8}\right)=4$. Now we cannot have $\ell\left(v_{6} v_{7}\right)=2$ as otherwise we would have $c_{\ell}\left(v_{7}\right)=c_{\ell}\left(v_{3}\right)=4$ while $v_{3}$ and $v_{7}$ share $v_{8}$ as a common neighbour. Thus $\ell\left(v_{6} v_{7}\right)=1$, and thus $c_{\ell}\left(v_{7}\right)=3$. Now, if $\ell\left(v_{5} v_{6}\right)=1$, then $v_{5}$ neighbours $v_{4}$ and $v_{6}$ which both have colour 2 , while, if $\ell\left(v_{5} v_{6}\right)=2$, then $v_{6}$ neighbours $v_{5}$ and $v_{7}$ which both have colour 3 . Thus with get a contradiction in all cases.

- Assume $\ell\left(v_{2} v_{3}\right)=2$. Then $c_{\ell}\left(v_{2}\right)=4$.

First, consider the case $\ell\left(v_{3} v_{4}\right)=\ell\left(v_{3} v_{8}\right)=1$. Then $c_{\ell}\left(v_{3}\right)=4$. Since $v_{3}$ neighbours both $v_{4}$ and $v_{8}$, we must have, say, $\ell\left(v_{4} v_{5}\right)=1$ and $\ell\left(v_{8} v_{7}\right)=2$, which yields $c_{\ell}\left(v_{4}\right)=2$ and $c_{\ell}\left(v_{8}\right)=3$. Now we cannot have $\ell\left(v_{6} v_{7}\right)=2$, as otherwise $v_{7}$ would have colour 4 just as $v_{3}$, and they share $v_{8}$ as a neighbour. So $\ell\left(v_{6} v_{7}\right)=1$, and $c_{\ell}\left(v_{7}\right)=3$. Now we get a contradiction no matter whether $v_{5} v_{6}$ is labelled 1 or 2 : in the first case, $v_{5}$ is a neighbour of both $v_{4}$ and $v_{6}$ which would have colour 2 , while, in the second case, $v_{6}$ is a neighbour of $v_{5}$ and $v_{7}$ which would both have colour 3 .

Second, consider the case $\ell\left(v_{3} v_{4}\right)=1$ and $\ell\left(v_{3} v_{8}\right)=2$. Then $c_{\ell}\left(v_{3}\right)=5$. Because $v_{3}$ neighbours $v_{2}$ and $v_{8}$, we must have $\ell\left(v_{8} v_{7}\right)=1$, which yields $c_{\ell}\left(v_{8}\right)=3$. Now, because $v_{3}$ neighbours $v_{4}$ and $v_{8}$, we must have $\ell\left(v_{4} v_{5}\right)=1$, which gives $c_{\ell}\left(v_{4}\right)=2$. Since $v_{6}$ shares a common neighbour with both $v_{4}$ and $v_{8}$, note that the edges incident to $v_{6}$ must be labelled to that $c_{\ell}\left(v_{6}\right)=4$. So the two edges must be labelled 2 , which yields $c_{\ell}\left(v_{5}\right)=c_{\ell}\left(v_{7}\right)=3$, a contradiction.

Third, consider the case $\ell\left(v_{3} v_{4}\right)=\ell\left(v_{3} v_{8}\right)=2$. Then $c_{\ell}\left(v_{3}\right)=6$. Because $v_{3}$ is a common neighbour of $v_{2}$ and $v_{4}$, and of $v_{2}$ and $v_{8}$, we must have $\ell\left(v_{4} v_{5}\right)=\ell\left(v_{8} v_{7}\right)=1$, which yields $c_{\ell}\left(v_{4}\right)=c_{\ell}\left(v_{8}\right)=3$, while $v_{3}$ is a common neighbour of $v_{4}$ and $v_{8}$, a contradiction.

We now need an infinite family of additional gadgets (see Figure 2 for an illustration). The $(1,2)$-gadget $G_{1,2}$ is a star on three vertices with two leaves $u, v$ and root $r$, where $r u$ and $r v$ are the two root edges, while, for the sake of consistency with what follows, $u$ and $v$ are called the subroots. Now, for every $k \geq 4$ with $k \equiv 1 \bmod 3$, the $(k, k+1)$-gadget $G_{k, k+1}$ is obtained by considering two copies $H_{1,2}, H_{1,2}^{\prime}$ of $G_{1,2}$, two copies of $H_{4,5}, H_{4,5}^{\prime}$, and similarly, for every $i<k$ with $i \equiv 1 \bmod 3$, two copies $H_{i, i+1}, H_{i, i+1}^{\prime}$ of $G_{i, i+1}$, identifying the roots of $H_{1,2}, H_{4,5}, \ldots, H_{k-3, k-2}$ to a single vertex $u$, identifying the roots of $H_{1,2}^{\prime}, H_{4,5}^{\prime}, \ldots, H_{k-3, k-2}^{\prime}$ to a single vertex $v$, and adding two edges $u r$ and $v r$ where $r$ is a new vertex. We call $r$ the root of $G_{k, k+1}$, the subroots are $u$ and $v$, while $r u$ and $r v$ are the two root edges. The properties of interest of these gadgets are the following.

Lemma 5.2. Let $k \geq 1$ with $k \equiv 1 \bmod 3$, and let $\ell$ be an injective 2 -labelling of the $(k, k+1)$-gadget $G_{k, k+1}$ with root $r$ and subroots $u$ and $v$. Then: 


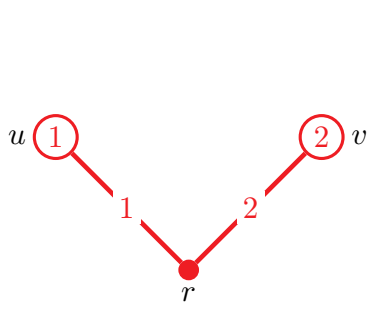

(a) $G_{1,2}$

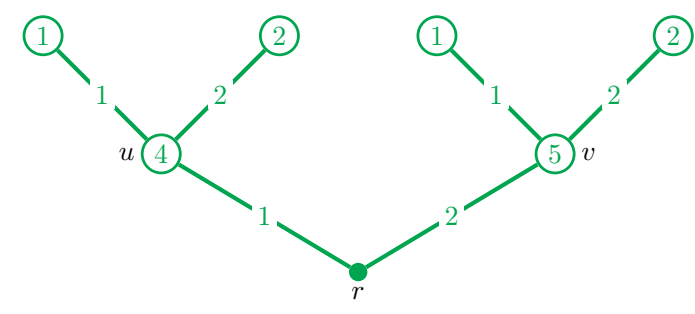

(b) $G_{4,5}$

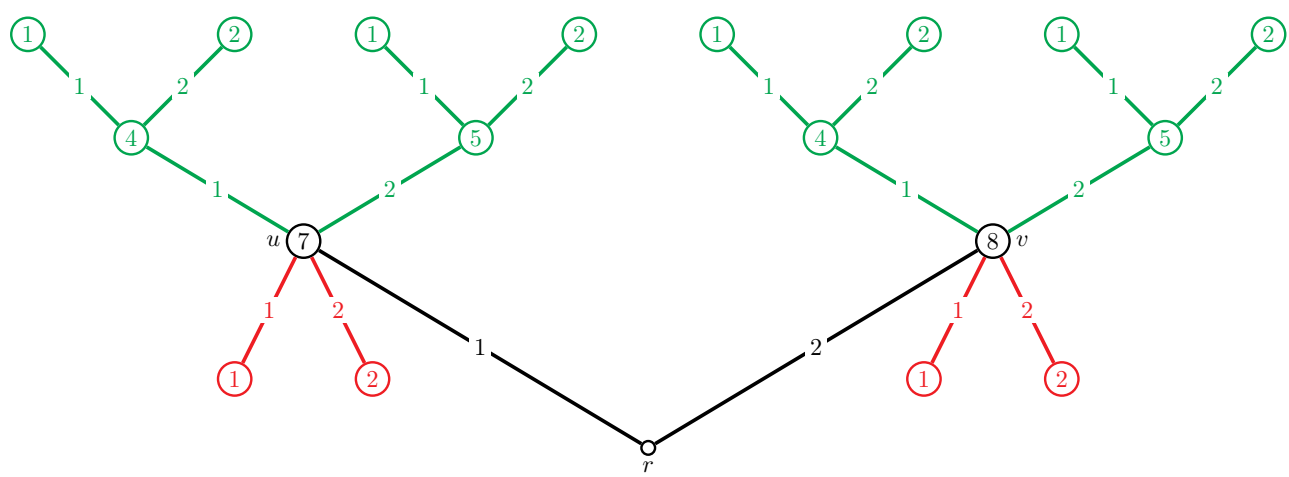

(c) $G_{7,8}$

Figure 2: Examples of $(k, k+1)$-gadgets, and their unique injective 2-labelling. An integer in a vertex indicates its colour by the depicted labelling.

- $\ell(r u)+\ell(r v)=3$;

- $\left\{c_{\ell}(u), c_{\ell}(v)\right\}=\{k, k+1\}$;

- $c_{\ell}(r)=3$;

- when $k>1, u$ is adjacent to a vertex with colour $x$, for every $x<k$ with $x \neq \equiv 0 \bmod 3$;

- when $k>1$, all vertices different from $u$ and $v$ have colour strictly less than $k$.

Proof. Note that the first item implies the third item. We prove the claim by induction on $k$. This is true for $k=1$ : because $r$ is a common neighbour of $u$ and $v$, and $d(u)=d(v)=1$, we must have $\ell(u r) \neq \ell(v r)$ so that $c_{\ell}(u) \neq c_{\ell}(v)$, which gives, say, $c_{\ell}(u)=1, c_{\ell}(v)=2$ and $c_{\ell}(r)=3$. Now assume the claim is true for all values of $k$ up to some $i \geq 1$, and consider the next step $k=i+3$. By the induction hypothesis, all the gadgets $H_{1,2}, \ldots, H_{k-3, k-2}$ attached at $u$, and similarly all the gadgets $H_{1,2}^{\prime}, \ldots, H_{k-3, k-2}^{\prime}$ attached at $v$ must be labelled by $\ell$ so that, for each $H_{j, j+1}$ (resp. $H_{j, j+1}^{\prime}$ ) of them, the two root edges incident to $u$ (resp. $v$ ) are labelled 1 and 2 , and $u$ (resp. $v$ ) neighbours vertices in $H_{j, j+1}$ with colour $j$ and $j+1$, while all other vertices of the gadget have colour less than $j$. Thus, the labelling of all these gadgets implies that the colour of $u$ and $v$ is at least $k-1$, while all other vertices have colour strictly less than $k$. Now, since $r$ is a common neighbour of $u$ and $v$, we must have $c_{\ell}(u) \neq c_{\ell}(v)$, and the only way to achieve this is to have, say, $\ell(u r)=1$ and $\ell(v r)=2$. This yields $c_{\ell}(u)=k$ and $c_{\ell}(v)=k+1$. Note that all conditions in the statement are met.

We are now ready to prove our main result.

Theorem 5.3. Given a graph $G$, it is $N P$-complete to decide whether $\chi_{\mathrm{i} \Sigma}(G) \leq 2$ holds. This remains true if $G$ is assumed bipartite.

Proof. The problem is clearly in NP. We prove its NP-hardness by reduction from Monotone CuBIC 1-IN-3 SAT, which is NP-hard [12]. An instance of this problem is a 3CNF formula $F$ with $n$ variables $x_{1}, \ldots, x_{n}$ and $m$ clauses $C_{1}, \ldots, C_{m}$, where each clause contains exactly three 
distinct variables (no negations), while every variable appears in exactly three distinct clauses. The question is whether $F$ can be 1-in-3 satisfied, i.e., whether there is a truth assignment to the variables such that every clause has exactly one true variable. From $F$, we construct, in polynomial time, a bipartite graph $G$ such that $F$ is 1-in-3 satisfiable if and only if $\chi_{\mathrm{i} \Sigma}(G) \leq 2$.

We start from $G$ being the cubic bipartite graph modelling the structure of $F$. That is, for every variable $x_{i}$ of $F$ we have a variable vertex $v_{i}$ in $G$, for every clause $C_{j}$ of $F$ we have a clause vertex $c_{j}$ in $G$, and whenever a variable $x_{i}$ belongs to a clause $C_{j}$ in $F$, we have a formula edge $v_{i} c_{j}$ in $G$.

We next consider every clause vertex of $G$ in turn. Let us consider $c_{1}$ first. We attach the root of a 1-gadget at $c_{1}$, as well as the roots of a $(13,14)$-gadget and of a $(16,17)$-gadget. Next consider $c_{2}$. We attach the root of a 1-gadget at $c_{2}$, as well as the roots of a $(13,14)$-gadget, of a $(16,17)$-gadget and of a $(19,20)$-gadget. The construction goes on like this for every $c_{j}$ : we attach the root of a 1-gadget at $c_{j}$, as well as the root of a $(k, k+1)$-gadget for every $k \in\{13, \ldots, 13+3 j\}$ with $k \equiv 1 \bmod 3$.

Finally we consider every variable vertex in turn. At $v_{1}$, we attach the root of a $(10,11)$-gadget. At $v_{2}$, we attach the roots of a $(10,11)$-gadget, of a $(13,14)$-gadget and of a $(16,17)$-gadget. At $v_{3}$, we attach the roots of a $(10,11)$-gadget, of a $(13,14)$-gadget, of a $(16,17)$-gadget, of a $(19,20)$ gadget and of a $(22,23)$-gadget. More generally, at every $v_{i}$, we attach the root of a $(k, k+1)$-gadget for every $k \in\{10, \ldots, 10+6(i-1)\}$ with $k \equiv 1 \bmod 3$.

Note that the construction of $G$ is achieved in polynomial time, and that $G$ is indeed bipartite. This is because we have started from a cubic bipartite graph (modelling the structure of $F$ ), and have only attached to the vertices some copies of $T$, which is bipartite, and some copies of gadgets $G_{k, k+1}$, which are trees. Also, note that there is no vertex neighbouring a clause vertex and a variable vertex.

Let $\ell$ be an injective 2-labelling of $G$. Recall that, by Lemma 5.1, all 1-gadgets attached to the clause vertices have their root edge labelled 1 , their subroot has colour at most 3 , and their subroot neighbours a vertex with a colour that can be at most 6 . By Lemma 5.2, all $(k, k+1)$-gadgets attached to the (clause and variable) vertices have their root edges labelled so that the sum of their labels is 3 , each of these $(k, k+1)$-gadgets has its subroots being adjacent to vertices with colour $k-3$ and $k-2$, and the root is potentially the only vertex of the whole gadget that has a colour being a multiple of 3 . By these remarks, we note that:

- For every clause vertex $c_{j}$, the labels of the incident root edges sum up to $3 j+4 \geq 10$. Since there are only three other incident edges (the formula ones), this means that $c_{\ell}\left(c_{j}\right)$ must lie in $\{3 j+7,3 j+8,3 j+9,3 j+10\}$. Furthermore, since, for every $10 \leq k \leq 13+3 j+1$ with $k \neq \equiv \bmod 3$, there is a vertex (subroot) adjacent to $c_{j}$ that itself neighbours a vertex (different from $c_{j}$ ) with colour $k$, this means that $c_{\ell}\left(c_{j}\right)$ must be $3 j+9$ (which is a multiple of 3), i.e., exactly one formula edge incident to $c_{j}$ must be labelled 1 while the other two incident edges must be labelled 2 .

- By those arguments, no two clause vertices $c_{j}$ and $c_{j^{\prime}}$ can get the same colour by $\ell$.

- For every variable vertex $v_{i}$, the labels of the incident root edges sum up to $6 i-3$. There are only three other incident edges (the formula ones), which means that $c_{\ell}\left(v_{i}\right)$ must lie in $\{6 i, 6 i+1,6 i+2,6 i+3\}$. Also, by construction, for every $7 \leq k \leq 10+6(i-1)+1$ with $k \neq 0 \bmod 3$, there is a subroot adjacent to $v_{i}$ which neighbours a vertex (different from $v_{i}$ ) with colour $k$. Then, $c_{\ell}\left(v_{i}\right)$ must be $6 i$ or $6 i+3$ (i.e., a multiple of 3 ). This happens when the remaining three formula edges incident to $v_{i}$ are all labelled 1 (for $6 i$ ), or are all labelled 2 (for $6 i+3)$.

- By these arguments, no two variable vertices $v_{i}$ and $v_{i^{\prime}}$ can have the same colour by $\ell$.

By all these arguments, we get that, so that $\ell$ raises no conflict, we must manage to label the formula edges so that 1 ) for every clause vertex there is exactly only one incident formula edge labelled 1, and 2) for every variable vertex all three incident formula edges are assigned the same label. The equivalence with 1-in-3 satisfying $F$ is then easy to see, by just considering that assigning 
label 1 (resp. 2) to the formula edge $v_{i} c_{j}$ means that variable $x_{i}$ brings truth value true (resp. false) to clause $C_{j}$. The first condition above models the fact that every clause is regarded satisfied only if it has exactly one true variable by a truth assignment. The second condition models the fact that, by a truth assignment, all variables bring the same truth value to all clauses containing it.

\section{Conclusion and perspectives}

In this work, we have introduced and investigated the notion of injective labellings, as a variant of the 1-2-3 Conjecture for injective colouring of graphs. Our guiding line was Conjecture 1.2 towards which we have provided several results. In particular, the first part of the conjecture holds for trees, for most cacti, and more generally for most graphs with injective chromatic number being equal to the maximum degree. We have also shown that determining $\chi_{\mathrm{i} \Sigma}(G)$ for a given bipartite graph $G$ is an NP-complete problem. We are very far, however, from fully understanding the problem.

Towards finding counterexamples to Conjecture 1.2, there are a few graph classes which could be legitimate candidates to consider. A first class could be the class of graphs $G$ with $\chi_{\mathrm{i}}(G)=|V(G)|$, which are, in some sense, the equivalent for injective colouring of complete graphs for proper colouring. We call these graphs injective-complete. It was shown in [7] that injective-complete graphs are exactly the graphs with diameter at most 2 in which every edge belongs to a triangle. Since all vertices of injective-complete graphs require different colours by an injective colouring, it might be that some of them require large labels to be labelled in an injective way. So we ask:

Question 6.1. Is Conjecture 1.2 true for injective-complete graphs?

We note that Question 6.1 is actually a weakening of the 1-2-3 Conjecture, since, by both an injective labelling and a proper labelling of an injective-complete graph, all adjacent vertices must receive different colours. In Corollary 6.4 below, we give a context in which Question 6.1 is positive.

Lemma 6.2. In every injective-complete graph, every cut-vertex is a dominating vertex.

Proof. Let $G$ be an injective-complete graph with a cut-vertex $v$. Then note that if a vertex $u$ from a component of $G-v$ is not adjacent to $v$, then $u$ is at distance more than 2 from the vertices in the other components. This implies that $G$ has diameter more than 2, a contradiction.

Lemma 6.3. For every graph $G$ of order at least 4 with a dominating vertex, we have $\chi_{\mathrm{i} \Sigma}(G) \leq$ $|V(G)|-1=\Delta(G)$.

Proof. Let $v$ be a dominating vertex of $G$. Consider the following $\Delta(G)$-labelling $\ell$ of $G$. For every edge $e$ not incident to $v$, we set $\ell(e)=1$. We now look at the current partial colour $s(u)$ of every vertex $u$ different from $v$, and we order the vertices $u_{1}, \ldots, u_{n-1}$ different from $v$ in such a way that $s\left(u_{i}\right) \leq s\left(u_{j}\right)$ whenever $i \leq j$. We achieve the construction of $\ell$ by setting $\ell\left(v u_{i}\right)=i$ for every $i \in\{1, \ldots, n-1\}$. Because we have $\ell\left(v u_{i}\right) \leq \ell\left(v u_{j}\right)$ whenever $i \leq j$, and also $s\left(u_{i}\right) \leq s\left(u_{j}\right)$, note that we also have $c_{\ell}\left(u_{i}\right)<c_{\ell}\left(u_{j}\right)$. Also, we have $c_{\ell}\left(u_{i}\right) \leq \Delta(G)+\Delta(G)-1=2 \Delta(G)-1$ for every $i \in\{1, \ldots, n-1\}$, while $c_{\ell}(v)=\sum_{i=1}^{\Delta(G)} i=\frac{\Delta(G)(\Delta(G)+1)}{2}$. Thus, $c_{\ell}(v)>c_{\ell}\left(u_{i}\right)$ since $\Delta(G) \geq 3$. This means that no two vertices have the same colour by $\ell$, which is thus injective.

Combining the previous two lemmas now gives the following.

Corollary 6.4. Conjecture 1.2 is true for every injective-complete graph of order at least 4 with a cut-vertex.

What makes the proof of Corollary 6.4 work is that the considered graphs have large maximum degree (compared to the number of vertices). We note however that injective-complete graphs, in general, can have much lower maximum degree. To see this is true, consider any graph $G$ with diameter at most 2 , choose $k \geq 2$ an even number, and let $H$ be the graph obtained from $G$ as follows. For every vertex $v$ of $G$, we add, in $H$, a set $S_{v}$ of $k$ new independent vertices. Then, for every edge $u v$ of $G$, in $H$ we completely join $S_{u}$ and $S_{v}$ (that is we add an edge joining every vertex of $S_{u}$ and every vertex of $S_{v}$ ). Finally, for every set $S_{v}$ of $H$, we add a perfect matching 


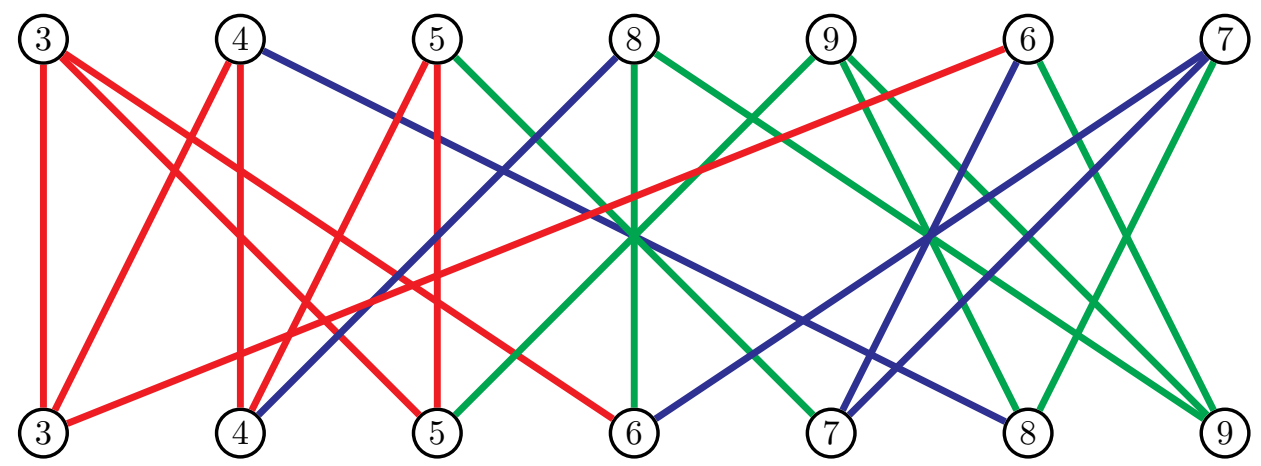

Figure 3: An injective 3-labelling of $\mathcal{P}_{3}$. Red edges are labelled 1, blue edges are labelled 2, and green edges are labelled 3 . An integer in a vertex indicates its colour.

(i.e., $k / 2$ independent edges) over the vertices of $S_{v}$. It is easy to check that $H$ has diameter 2, and every edge is contained in a triangle; thus $H$ is injective-complete. However, we note that $\Delta(H)=k \Delta(G)+1$, while $|V(H)|=k|V(G)|$. For instance, if we take the Petersen graph as $G$, then the graph $H$ we get via this construction verifies $\Delta(H)=3 k+1$ and $|V(H)|=10 k$.

Regarding Conjecture 1.2, another interesting class of graphs to consider could be that of incidence graphs of projective planes, which are exactly the graphs with arbitrary maximum degree $\Delta$ for which the injective chromatic number is equal to the maximum value in theory, i.e., $\Delta(\Delta-$ $1)+1$, see [7. Every such graph $\mathcal{P}_{\Delta}$ is the bipartite graph obtained from the projective plane of order $\Delta$, by having vertices in one part corresponding to points, vertices in the other part corresponding to lines, and in which an edge indicates that some point lies on some line. As a result, $\mathcal{P}_{\Delta}$ is $\Delta$-regular, every two vertices in a same partite set are joined by a path of length 2 , and every partite set has cardinality $\Delta(\Delta-1)+1$.

Question 6.5. Is Conjecture 1.2 true for incidence graphs of projective planes?

We note that for every such graph $\mathcal{P}_{\Delta}$, the possible colours for the vertices by a $\Delta$-labelling range in $\left\{\Delta, \ldots, \Delta^{2}\right\}$, which is a set of cardinality $\Delta^{2}-\Delta+1$. Thus, in an injective $\Delta$-labelling of $\mathcal{P}_{\Delta}$, for every partite set the set of the colours of its vertices should be exactly $\left\{\Delta, \ldots, \Delta^{2}\right\}$. We were unable to find a labelling scheme guaranteeing this for all $\mathcal{P}_{\Delta}$ 's. However, as depicted in Figure 3, such a 3-labelling exists for $\mathcal{P}_{3}$.

\section{References}

[1] Y. Alavi, G. Chartrand, F.R.K. Chung, P. Erdős, R.L. Graham, O.R. Oellermann. How to define an irregular graph. Journal of Graph Theory, 11(2):235-249, 1987.

[2] O. Baudon, J. Bensmail, H. Hocquard, M. Senhaji, É. Sopena. Edge Weights and Vertex Colours: Minimizing Sum Count. Discrete Applied Mathematics, 270:13-24, 2019.

[3] O. Baudon, J. Bensmail, J. Przybyło, M. Woźniak. On decomposing regular graphs into locally irregular subgraphs. European Journal of Combinatorics, 49:90-104, 2015.

[4] J. Bensmail, F. Mc Inerney, K. Szabo Lyngsie. On $\{a, b\}$-edge-weightings of bipartite graphs with odd $a, b$. Accepted for publication in Discussiones Mathematicae Graph Theory.

[5] G.J. Chang, C. Lu, J. Wu, Q. Yu. Vertex-coloring edge-weightings of graphs. Taiwanese Journal of Mathematics, 15(4):1807-1813, 2011.

[6] A. Dudek, D. Wajc. On the complexity of vertex-coloring edge-weightings. Discrete Mathematics Theoretical Computer Science, 13(3):45-50, 2011.

[7] G. Hahn, J. Kratochvíl, Širáň, D. Sotteau. On the injective chromatic number of graphs. Discrete Mathematics, 256:179-192, 2002. 
[8] M. Kalkowski, M. Karoński, F. Pfender. Vertex-coloring edge-weightings: towards the 1-2-3 Conjecture. Journal of Combinatorial Theory, Series B, 100:347-349, 2010.

[9] M. Karoński, T. Łuczak, A. Thomason. Edge weights and vertex colours. Journal of Combinatorial Theory, Series B, 91:151-157, 2004.

[10] H. Lu, Q. Yu, C.-Q. Zhang. Vertex-colouring 2-edge-weightings of graphs. European Journal of Combinatorics, 32:21-27, 2011.

[11] B. Lužar, R. Škrekovski. Counterexamples to a conjecture on injective colorings. Ars Mathematica Contemporanae, 8:291-295, 2015.

[12] C. Moore, J.M. Robson. Hard Tiling Problems with Simple Tiles. Discrete and Computational Geometry, 26(4):573-590, 2001.

[13] J. Petersen, Die Theorie der regulagğaren Graphen. Acta Mathematica, 15:193-220, 1891.

[14] B. Seamone. The 1-2-3 Conjecture and related problems: a survey. Technical report, available online at http://arxiv.org/abs/1211.5122, 2012.

[15] K. Szabo Lyngsie. On neighbour sum-distinguishing $\{0,1\}$-weightings of bipartite graphs. Discrete Mathematics and Theoretical Computer Science, 20(1), 2018, \#21.

[16] C. Thomassen, Y. Wu, C.-Q. Zhang. The 3-flow conjecture, factors modulo $k$, and the 1-2-3conjecture. Journal of Combinatorial Theory, Series B, 121:308-325, 2016. 